\title{
ITERATED PATH INTEGRALS ${ }^{1}$
}

\author{
BY KUO-TSAI CHEN
}

The classical calculus of variation is a critical point theory of certain differentiable functions (or functionals) on a smooth or piecewise smooth path space, whose differentiable structure is defined implicitly. Because of the importance of path spaces to analysis, geometry and other fields, it is desirable to develop a geometric integration theory or a de Rham theory for path spaces. Having in mind this general goal, we are going to consider a large class of path space differential forms, which can be constructed from usual differential forms by a method of iterated integration.

Recall that the Poincare lemma is proved through a process of integration, which converts every closed $p$-form $w$ on a manifold $M$ locally to a $(p-1)$ form. The same process can be used to obtain a $(p-1)$-form $\int w$ defined globally on the total smooth space $P(M)$ of $M$. More generally, given forms $w_{1}, \ldots, w_{r}$ on $M$, we may repeat the integration process $r$ times in an appropriate manner and obtain a differential form $\int w_{1} \cdots w_{r}$ on $P(M)$ of degree $-r+\sum_{1 \leqslant i \leqslant r} \operatorname{deg} w_{i}$. Such path space differential forms and their linear combinations will be called iterated (path) integrals.

Our objective is to determine the geometrical significance of such iterated integrals. It turns out that they play a surprisingly interesting role in relating analysis on a manifold (or differentiable space) to the homology of its path spaces. For example, Theorem 2.3.1 implies that the real loop space cohomology of a simply connected compact manifold is isomorphic to the cohomology of the complex of iterated integrals as differential forms on the smooth loop space $\Omega M$. Iterated integrals are path space differential forms which permit further integration. They also provide analytic interpretation or realization of algebraic topological notions such as bar constructions [34], [12], Eilenberg-Moore spectral sequences [60], [61], Massey products [46], [51] and loop space cohomology classes of Kraines [47].

Our de Rham theoretical approach also produces computational tools, which have the advantage of dealing with commutative differential graded algebras of relatively simple structure. Examples will illustrate how our

An expanded version of an address delivered before the St. Louis meeting of the Society on April 11, 1975; received by the editors April 5, 1976.

AMS (MOS) subject classifications (1970). Primary 58A99, 55D35; Secondary 53B15, 53C65, $55 \mathrm{H} 20,49 \mathrm{~F} 05$.

Key words and phrases. Differentiable spaces, differential forms, path space calculus, loop space homplogy, fundamental groups, twisting cochain, bar and cobar constructions, Eilenberg-Moore spectral sequence, fibration.

${ }^{1}$ Supported in part by National Science Foundation under MPS 72-04777. 
methods can be used in the computation of homology and cohomology of loop spaces and other path spaces.

We adopt a simple but very general notion of differentiable spaces, which will be effectively and indispensably used.

D. Sullivan has an elegant and efficient theory of minimal models, which can be regarded as a de Rham homotopy theory. Though his theory and ours are differently motivated, their algebraic topological aspects are intimately related by the fact that rational homotopy and the rational loop space homology determine each other at least in the simply connected case according to a theorem of Milnor and Moore [54]. On the analytical level, a precise relation between the two theories has yet to be satisfactorily established. For Sullivan's theory, see [31], [37] and [66].

Iterated integrals of differential 1 -forms are rather obvious generalizations of line integrals in calculus and have long existed. See $\S 1.1$ for background material.

The integral used by J. H. C. Whitehead [70] for Hopf invariants can be taken as a kind of twice iterated integral. A homotopy group theoretical approach to iterated integrals was given in [22]. It has been abandoned in favor of the present path space approach, which is better adapted to analysis.

Chapter I covers the analytic aspect of this work. Chapter II is devoted mainly to establishing de Rham type theorems. Chapter III describes a method of computing loop space homology. The method involves a special kind of generalized linear connections satisfying a twisting cochain condition. Chapter IV relates the bar construction to iterated integrals and presents a de Rham type theorem for pullbacks of the free path fibration. Among such pullbacks are various subspaces of the free path space. The Appendix treats the cobar construction for differentiable spaces and gives a proof of Adams' theorem [1], which is instrumental in this work. Generally speaking, material in the first three chapters summarizes, improves or supplements previous works, while the last chapter contains mostly new material, which is included to indicate both the scope and the future prospect of this work.

Our presentation will be in terms of smooth paths instead of piecewise paths as in [24]. The symbol $I$ will denote the unit interval. Every manifold will be $C^{\infty}$ and paracompact. The symbol $k$ denotes the field of real (or complex) numbers. All vector spaces, linear maps and tensor products will be over $k$ unless otherwise stated. By a differential graded algebra $A$, we shall mean a graded algebra with a differential of degree 1 such that $A^{p}=0$ for $p<0$ and $1 \in A^{0}$. By $A$ being commutative, we shall mean that $u v=$ $(-1)^{p q} v u, \forall u \in A^{p}, v \in A^{q}, p, q \geqslant 0$.

I am indebted to R. Bott, W. S. Massey and J. D. Stasheff for their valuable suggestions in connection with the loop space homology and the cobar construction. I also wish to take this opportunity to thank and Institute for Advanced Study for the stimulating environment during my visits, which contributed a great deal to the subsequent progress of this work.

\section{Chapter I. Path SPace Calculus}

After introducing iterated integrals of 1 -forms on a manifold, we proceed to treat differentiable spaces, define the notion of iterated integrals in general 
and discuss their properties. A substantial part of the material in this chapter can be found in [24].

1.1. Generalization of line integrals by iteration. Let us first consider the case of the real line $R$. A 1 -form on $R$ can be written as $f(t) d t$. Let $\int_{a}^{b} f(t) d t$ denote the usual integral. For $r>1$, define inductively

$$
\int_{a}^{b} f_{1}(t) d t \cdot \cdots f_{r}(t) d t=\int_{a}^{b}\left(\int_{a}^{t} f_{1}(\tau) d \tau \cdots f_{r-1}(\tau) d \tau\right) f_{r}(t) d t
$$

When $r=0$, set the integral to be 1 . Such iterated integrals occur in the Picard's approximation of a system of linear ordinary differential equations.

If the real line $R$ is replaced by the complex plane $C$ and if $f_{1}(t), \ldots, f_{r}(t)$ are holomorphic functions of a complex variable $t$, then the iterated integral (1.1.1) again makes sense. Paršin has considered iterated integrals of this type on Riemann surfaces [56].

More generally, let $w_{1}, w_{2}, \ldots$ be 1 -forms on a manifold $M$ and let $\gamma$ : $I \rightarrow M$ be a smooth path. Write

$$
\gamma^{*} w_{i}=f_{i}(t) d t
$$

and define

$$
\int_{\gamma} w_{1} \cdots w_{r}=\int_{0}^{1} f_{1}(t) d t \cdots f_{r}(t) d t
$$

which will be referred to as an iterated line integral. There are sufficiently many such iterated line integrals to separate essentially distinct paths in the manifold $M$. We state the following corollary of Theorem 4.2 [15]:

Let $w_{1}, \ldots, w_{m}$ be 1 -forms on a manifold $M$, which span the cotangent space at every point of $M$. Then two regular $C^{\infty}$ paths $\alpha$ and $\beta$ having a common initial point in $M$ differ by a parametrization if and only if $\int_{\alpha} w_{i_{1}} \cdots w_{i_{r}}=\int_{\beta} w_{i_{1}} \cdots w_{i_{r}}$ for $r \geqslant 1, i_{1}, \ldots, i_{r}=1, \ldots, m$.

Various aspects of iterated line integrals were considered in [13]-[22], [25], [26], [30]. Ree observed the shuffle multiplication of iterated line integrals in [57]. For other related work, see Johnson [42] and Asada [6].

An obvious problem at this point is to study homotopy invariant (iterated line) integrals, namely, linear combinations of iterated line integrals whose value along each path depends only on the path homotopy class.

A simple line integral $\int w$ is homotopy invariant if and only if $w$ is a closed 1-form. There are many other examples of homotopy invariant integrals. (See [21].) If $w_{1}$ and $w_{2}$ are closed 1-forms such that $w_{1} \wedge w_{2}+d w_{12}=0$ for some 1 -form $w_{12}$, then the iterated line integral

$$
\int w_{1} w_{2}+w_{12} \stackrel{\text { def }}{=} \int w_{1} w_{2}+\int w_{12}
$$

is homotopy invariant. This can be verified by lifting the integral to the universal covering manifold of $M$.

EXAMPLE 1.1.1. Let $L_{1}$ and $L_{2}$ be the two circles in $R^{3}$ respectively given by the equations 


$$
y^{2}+z^{2}=1, \quad x=0
$$

and

$$
z^{2}+x^{2}=4, \quad y=0
$$

Let $w_{i}, i=1,2$, be a closed 1 -form on $R^{3}-L_{i}$ representing a generator of the integral cohomology group $H^{1}\left(R^{3}-L_{i}\right)$ via integration. Since $L_{1}$ and $L_{2}$ are unlinked, $w_{1} \wedge w_{2}$ must be exact on $M=R^{3}-L_{1} \cup L_{2}$ so that $w_{1} \wedge w_{2}+$ $d w_{12}=0$ for some 1 -form $w_{12}$, and the integral (1.1.2) is homotopy invariant in $M$.

Let $\gamma$ be a loop in $M$ which traces $n$ times around the ellipse:

$$
x^{2} / a^{2}+y^{2} / b^{2}=1, \quad z=0,
$$

with $a>3$ and $0<b<1$. It can be verified that $\int_{\gamma} w_{1}=\int_{\gamma} w_{2}=0$ and $\int_{\gamma} w_{1} w_{2}+w_{12}= \pm n$. The integer $n$ represents a higher order linking number. Such higher order linking numbers have been defined through Massey products in [50].

An iterated line integral can be taken as a functional in the sense of the calculus of variation, and a homotopy invariant integral is then a locally constant functional whose calculus-variational differential is everywhere zero. We are now interested in a differential formula for iterated line integrals. It will happen that the differential of an iterated line integral is again an iterated integral, which, of course, involves 2 -forms.

1.2. Differentiable spaces. There are two ways to define differentiable structures. The first is through using charts, which identify open sets with euclidean open sets, whose differentiable structure is known. The second is by mapping euclidean sets (of various dimensions) into the manifold in consideration. The latter approach is implicit in classical analysis in terms of parametrization. J. W. Smith [62] has defined and studied general differentiable spaces from this approach. Using the same approach, we are going to adopt a notion of differentiable spaces, for which no topology is needed.

The symbols $U, U^{\prime}, U_{i}, \ldots$ will denote convex sets. All convex sets will be finite dimensional. They will serve as models, i.e. sets whose differentiable structure is known.

If $\operatorname{dim} U=n, U$ can be linearly embedded in $R^{n}$, which provides $U$ with coordinates $\xi=\left(\xi^{1}, \ldots, \xi^{n}\right)$. Since $U$ has a nonempty interior in $R^{n}$, the de Rham complex $\Lambda(U)$ on $U$ is well defined, and every ( $k$-valued) $p$-form on $U$ can be written as

$$
\sum a_{i_{1}} \cdots i_{p}(\xi) d \xi^{i_{1}} \wedge \cdots \wedge d \xi^{i_{p}}
$$

with $a$ 's being ( $k$-valued) $C^{\infty}$ functions on $U$.

DEFINITION 1.2.1. A differentiable space $M$ is a set equipped with a family of set maps called plots, which satisfy the following conditions:

(a) Every plot is a map of the type $U \rightarrow M$, where $\operatorname{dim} U$ can be arbitrary.

(b) If $\phi: U \rightarrow M$ is a plot and if $\theta: U^{\prime} \rightarrow U$ is a $C^{\infty}$ map, then $\phi \circ \theta$ is a plot.

(c) Every constant map from a convex set to $M$ is a plot. 
(d) Let $\phi: U \rightarrow M$ be a set map. If $\left\{U_{i}\right\}$ is an open covering of $U$ and if each restriction $\phi \mid U_{i}$ is a plot, then $\phi$ itself is a plot.

Definition 1.2.2. A $p$-form on a differentiable space $M$ is a rule that assigns to each plot $\phi: U \rightarrow M$ a $p$-form $w_{\phi}$ on $U$ satisfying the condition: If $\theta: U^{\prime} \rightarrow U$ is a $C^{\infty}$ map, then

$$
w_{\phi \theta}=\theta^{*} w_{\phi} .
$$

Define the addition, the $k$-action, the exterior multiplication and the exterior differentiation through the formulas: $\left(w_{1}+w_{2}\right)_{\phi}=w_{1 \phi}+w_{2 \phi} ;(c w)_{\phi}$ $=c w_{\phi} ;\left(w_{1} \wedge w_{2}\right)_{\phi}=w_{1 \phi} \wedge w_{2 \phi}$ and $(d w)_{\phi}=d w_{\phi}$. Thus we obtain a commutative differential graded algebra $\Lambda(M)=\left\{\Lambda^{p}(M)\right\}$, which will be called the de Rham complex of the differentiable space $M$. We call

$$
H_{D R}^{*}(M) \stackrel{\text { def }}{=} H(\Lambda(M))
$$

the de Rham cohomology of $M$.

EXAMPLE 1.2.1. Every manifold $M$ (with or without boundary) is a differentiable space, whose plots are $C^{\infty}$ maps of the type $\phi: U \rightarrow M$. A usual $p$-form $w$ on the manifold $M$ is identified with the $p$-form $w$ on the differentiable space $M$ by setting $w_{\phi}=\phi^{*} w$ for every plot $\phi$.

EXAMPLE 1.2.2. Every subset $S$ of a differentiable space $M$ is a differentiable space, whose plots are maps $\psi$ such that $U \stackrel{\psi}{\rightarrow} S \subset M$ is a plot of $M$. (We shall call the differentiable space $S$ a differentiable subspace of $M$.) The de Rham complex $\Lambda(S)$ reflects the richness of the differentiable structure of $S$. For example, if $S$ is the set of rational numbers, then, as a differentiable subspace of the real line, $S$ has a de Rham complex with $\Lambda^{p}(S)=0$ for $p>0$, for every plot of $S$ must be a constant map.

Definition 1.2.3. Let $M$ and $M^{\prime}$ be differentiable spaces. A set map $f$ : $M \rightarrow M^{\prime}$ is a differentiable map if, for every plot $\phi$ of $M, f \phi$ is a plot of $M^{\prime}$.

Both homomorphisms $\Lambda\left(M^{\prime}\right) \rightarrow \Lambda(M)$ and $H_{D R}^{*}\left(M^{\prime}\right) \rightarrow H_{D R}^{*}(M)$ induced by a differentiable map $f$ will be denoted by $f^{*}$ when there is no ambiguity.

Proposition 1.2.1. If $f: M \rightarrow M^{\prime}$ is a constant map, then $f^{*}: \Lambda^{p}\left(M^{\prime}\right) \rightarrow$ $\Lambda^{p}(M), p>0$, are trivial.

This assertion follows from the fact that $f$ factors through the singleton differentiable space $\{0\}$, which has a trivial de Rham complex.

Definition 1.2.4. A predifferentiable space $M$ is a set equipped with a family of maps of the type $U \rightarrow M$ also called plots, which satisfies only the conditions (a), (b), and (c) of Definition 1.2.1.

Differential forms on a predifferentiable space can be defined in the same way as for a differentiable space.

Every predifferentiable space $M$ has an associated differentiable space structure, whose plots are set maps of the type $\phi: U \rightarrow M$ such that there exists an open covering $\left\{U_{i}\right\}$ of $U$ with each restriction $\phi \mid U_{i}$ being a plot of the predifferentiable space $M$. It is easy to verify that $M$, as a differentiable space, is well defined and that the de Rham complex of the predifferentiable space $M$ coincides with that of the differentiable space $M$.

Sometimes it is more convenient to define a differentiable space $M$ through 
a predifferentiable space in the above manner. We shall call such a predifferentiable space a defining predifferentiable space of $M$.

Hereafter $M$ will denote a differentiable space.

\subsection{Integration and chain complexes.}

Definition 1.3.1. A plot $\phi: U \rightarrow M$ of a differentiable space $M$ is compact if $U$ is compact.

If $w$ is a $p$-form on $M$ and if $\phi$ is compact, define $\int_{\phi} w=\int_{U} w_{\phi}$ when $\operatorname{dim} U=p$ and $\int_{\phi} w=0$ otherwise. We shall often write $\langle w, \phi\rangle=\int_{\phi} w$.

Definition 1.3.2. Let $\phi_{i}: U \rightarrow M, i=1, \ldots, l$, be plots on a common convex set $U$. A linear combination

$$
c=\sum_{1<i<l} n_{i} \phi_{i}, \quad n_{i} \in Z,
$$

will be called a plot chain of $M$ on $U$.

If $w$ is a differential form on $M$, define $w_{c}=\sum n_{i} w_{\phi_{i}}$. If $U$ is compact, define $\langle w, c\rangle=\int_{U} w_{c}$.

An $n$-simplex of $M$ is a plot of the type $\sigma: \Delta^{n} \rightarrow M$, where $\Delta^{n}$ is the standard $n$-simplex. Define the boundary $\partial \sigma$ in the usual way. We have $\langle d w, \sigma\rangle=\langle w, \partial \sigma\rangle$. Denote by $\Delta(M)=\left\{\Delta^{n}(M)\right\}$ the free chain complex on simplices of $M$. Then $\Delta^{n}(M)$ consists of plot chains of $M$ on $\Delta^{n}$. Write $H_{*}(M)=H(\Delta(M))$, which will be referred to as the homology of the differentiable space $M$.

An $n$-cube of $M$ is a plot of the type $\phi: I^{n} \rightarrow M$. An $n$-cube $\phi, n \geqslant 1$, is said to be degenerate if $\phi\left(\xi^{1}, \ldots, \xi^{n}\right)$ is independent of the first coordinate $\xi^{n}$.

Let $\lambda_{i}^{\varepsilon}: I^{n-1} \rightarrow I^{n}, 1 \leqslant i \leqslant n, \varepsilon=0,1$, be given by

$$
\left(\xi^{1}, \ldots, \hat{\xi}^{i}, \ldots, \xi^{n}\right) \mapsto\left(\xi^{1}, \ldots, \xi^{i-1}, \varepsilon, \xi^{i+1}, \ldots, \xi^{n}\right) .
$$

If $\phi$ is an $n$-cube of $M$, define

$$
\partial \phi=\sum_{1<i<n}(-1)^{i}\left(\phi \lambda_{i}^{0}-\phi \lambda_{i}^{1}\right)
$$

which is a plot chain of $M$ on $I^{n-1}$.

Denote by $C_{*}^{\prime}(M)$ the free chain complex on cubes of $M$ with the boundary operator $(1.3 .1)$ and by $C_{*}(M)$ the quotient of $C_{*}^{\prime}(M)$ over the subcomplex spanned by all degenerate cubes of $M$. We shall call $C_{*}(M)$ the normalized cubical chain complex of $M$. There is a natural isomorphism $H\left(C_{*}(M)\right) \approx H_{*}(M)$ which can be proved by an acyclic model argument. See [40] and [41] for further detail on the cubical homology.

Every topological space can be regarded as a differentiable space whose plots are continuous maps. If $X$ is a topological space, then the chain complex $\Delta(X)\left(\right.$ resp. $\left.C_{*}(X)\right)$ in the singular sense coincides with that in the differentiable sense.

Definition 1.3.3. A topological differential space $M$ is a differentiable space as well as a topological space (which will be denoted by ${ }_{T} M$ in order to distinguish it from the differentiable space $M$ ) such that every plot of $M$ is continuous with respect to the topology. 
Manifolds are topological differentiable spaces. So are most differentiable spaces we are going to consider.

For a topological differentiable space $M$, plots of $M$ will be called smooth plots while those of ${ }_{T} M$ will be called continuous plots. The same distinction applies to simplices, cubes, maps, etc.

The next lemma gives a sufficient condition for the smooth and continuous homologies to be identical.

Lemma 1.3.1. Let $M$ be a topological differentiable space such that, for every continuous simplex $\sigma: \Delta^{n} \rightarrow_{T} M$ with smooth $(n-1)$-faces, $n \geqslant 1$, there exists a continuous homotopy

$$
h: \Delta^{n} \times I \rightarrow_{T} M
$$

relative to the boundary $\dot{\Delta}^{n}$ of $\Delta^{n}$ from $\sigma$ to a smooth $n$-simplex of $M$. Then the canonical map $M \rightarrow_{T} M$ induces a chain equivalence

$$
\Delta(M) \rightarrow \Delta\left({ }_{T} M\right)
$$

as well as a chain equivalence $\Delta(M)_{x_{0}} \rightarrow \Delta\left({ }_{T} M\right)_{x_{0}}$, where $\Delta(M)_{x_{0}}$ denotes the subcomplex of $\Delta(M)$ spanned by simplices whose vertices are at a given base point $x_{0} \in M$.

This lemma can be verified by a standard argument. For manifolds, the above chain equivalences have been proved in [33].

1.4. The Poincaré operators. If $M_{1}$ and $M_{2}$ are differentiable spaces, then $M_{1} \times M_{2}$ is a differentiable space, which has a defining predifferentiable space whose plots are maps $\phi_{1} \times \phi_{2}: U_{1} \times U_{2} \rightarrow M_{1} \times M_{2}$ such that $\phi_{1}$ and $\phi_{2}$ are plots of $M_{1}$ and $M_{2}$ respectively.

Definition 1.4.1. Let $M^{\prime}$ be a differentiable space. A $\Lambda^{p}\left(M^{\prime}\right)$-valued function $u$ on $I$ is an element of $\Lambda^{p}\left(I \times M^{\prime}\right)$ such that, for every plot $\psi$ : $U \rightarrow M^{\prime}$, the $p$-form $u_{1 \times \psi}$ is of the type

$$
\sum a_{i_{1} \cdots i_{p}}(t, \xi) d \xi^{i_{1}} \wedge \cdots \wedge d \xi^{i_{p}}
$$

where $1=1_{I}$ denotes the identity map of $I$ and $\xi=\left(\xi^{1}, \ldots, \xi^{n}\right)$ denotes the coordinates of $U$.

For example, every element of $\Lambda^{p}\left(I \times M^{\prime}\right)$ that comes from $\Lambda^{p}\left(M^{\prime}\right)$ is a $\Lambda^{p}\left(M^{\prime}\right)$-valued function on $I$.

We shall of ten use $u=u(t)$ to denote a $\Lambda^{p}\left(M^{\prime}\right)$-valued function on $I$. Thus for $t_{0} \in I, u\left(t_{0}\right)$ is the image of $u$ under the homomorphism induced by the inclusion $M^{\prime}=\left\{t_{0}\right\} \times M^{\prime} \subset I \times M^{\prime}$.

If $u$ is a $\Lambda^{p}\left(M^{\prime}\right)$-valued function on $I$, define

$$
\int_{a}^{b} u d t \in \Lambda^{p}\left(M^{\prime}\right), \quad a, b \in I
$$

such that

$$
\left(\int_{a}^{b} u d t\right)_{\psi}=\sum\left(\int_{a}^{b} a_{i_{1} \cdots i_{p}}(t, \xi) d t\right) d \xi^{i_{1}} \wedge \cdots \wedge d \xi^{i_{p}}
$$

Similarly define the $\Lambda^{p}\left(M^{\prime}\right)$-valued function $\partial u / \partial t$ on $I$ such that $(\partial u / \partial t)_{1 \times \psi}$ is obtained by differentiating the coefficients of $u_{1 \times \psi}$ with respect to $t$. 
The 1 -form $d t$ is well defined on $I \times M^{\prime}$. Every $p$-form $v$ on $I \times M^{\prime}$ can be uniquely written as

$$
v=d t \wedge v^{\prime}+v^{\prime \prime}
$$

where $v^{\prime}$ and $v^{\prime \prime}$ are respectively $\Lambda^{p-1}\left(M^{\prime}\right)$ - and $\Lambda^{p}\left(M^{\prime}\right)$-valued functions on $I$. When $p=0$, set $v^{\prime}=0$.

We shall write $\left.v^{\prime}=(\partial / \partial t)\right\lrcorner v$, which coincides with the usual notation for an interior product in the case where $M$ is a manifold.

Define a (differentiable) homotopy between differentiable maps in the obvious way.

Lemma 1.4.1. Let $f_{0}, f_{1}: M^{\prime} \rightarrow M$ be differentiable maps. Then every homotopy $F: I \times M^{\prime} \rightarrow M$ from $f_{0}$ to $f_{1}$ induces a chain homotopy

$$
\int_{F}: \Lambda(M) \rightarrow \Lambda\left(M^{\prime}\right)
$$

given by $\left.\left.w \mapsto \int_{0}^{1}(\partial / \partial t)\right\lrcorner F^{*} w\right) d t$ such that

$$
d \int_{F}+\int_{F} d=f_{1}^{*}-f_{0}^{*}
$$

PROOF. Let $d_{M^{\prime}}$ denote the exterior differential in $M^{\prime}$. If $w \in \Lambda^{p}(M)$ and $F^{*} w=v=d t \wedge v^{\prime}+v^{\prime \prime}$, then

$$
\begin{aligned}
d \int_{F} w+\int_{F} d w & =\int_{0}^{1} d_{M^{\prime}} v^{\prime} d t+\int_{0}^{1}\left(-d_{M^{\prime}} v^{\prime}+\frac{\partial}{\partial t} v^{\prime \prime}\right) d t \\
& =v^{\prime \prime}(1)-v^{\prime \prime}(0)=f_{1}^{*} w-f_{0}^{*} w .
\end{aligned}
$$

REMARK. In the special case where $M=M^{\prime}$ is a convex set in $R^{n}$ and $F$ representing a contraction, the above lemma is the usual Poincare lemma. Therefore we call $\int_{F}$ the Poincaré operator of the homotopy $F$.

1.5. Iterated integrals. By a path in $M$, we mean a plot of the type $\gamma$ : $I \rightarrow M$. Denote by $P(M)$ the set of all paths in $M$. For every set map $\alpha$ : $U \rightarrow P(M)$, the suspension map of $\alpha$ is defined to be the map

$$
\phi_{\alpha}: I \times U \rightarrow M
$$

given by $(t, \xi) \mapsto \alpha(\xi)(t)$. Then $P(M)$ is a differentiable space having a defining predifferentiable space whose plots are maps of the type $\alpha: U \rightarrow$ $P(M)$ such that its suspension map $\phi_{\alpha}$ is a plot of $M$.

For every $t \in I$, there is a differentiable map $p_{t}: P(M) \rightarrow M$ given by $\gamma \mapsto \gamma(t)$. In particular, the maps $p_{0}$ and $p_{1}$ assign each path to its initial and end points respectively.

Let $\gamma^{t}$ be the path such that $\gamma^{\prime}(\tau)=\gamma(t \tau)$. Denote by $\tilde{p}_{t}: P(M) \rightarrow P(M)$ the differentiable maps given by $\gamma \mapsto \gamma^{\prime}$.

Denote by $\eta_{x}$ the constant path at $x$ in $M$. Denote by $\eta: M \rightarrow P(M)$ the canonical differentiable map given by $x \mapsto \eta_{x}$. Then there is a homotopy

$$
I \times P(M) \rightarrow P(M)
$$

from $\eta \circ p_{0}$ to the identity map $1_{P(M)}$ given by $(t, \gamma) \mapsto \gamma^{\prime}$. This is the homotopy obtained by contracting each path along itself. Denote by 


$$
\int^{\prime}: \Lambda(P(M)) \rightarrow \Lambda(P(M))
$$

the resulting Poincaré operator.

Lemma 1.5.1. Let $F$ denote the homotopy (1.5.2). If $v \in \Lambda^{p}(P(M))$, then $u=F^{*} \int^{\prime} v$ is a $\Lambda^{p-1}(P(M))$-valued function on $I$, and

$$
\left.u(s)=\int_{0}^{s}\left(\frac{\partial}{\partial t}\right\lrcorner F^{*} v\right) d t, \quad s \in I .
$$

Proof. For every plot $\alpha: U \rightarrow P(M)$, set $\alpha^{\prime}=F \circ\left(1_{I} \times \alpha\right)$ and $\alpha^{\prime \prime}=$ $F \circ\left(1, \times \alpha^{\prime}\right): I \times I \times U \rightarrow P(M)$, which is given by $\alpha^{\prime \prime}(t, s, \xi)(\tau)=\alpha(\xi)(t s \tau)$ and can be factorized as

$$
I \times I \times U \stackrel{\mu \times 1_{U}}{\rightarrow} I \times U \stackrel{\alpha^{\prime}}{\rightarrow} P(M)
$$

where $\mu: I \times I \rightarrow I$ is such that $(t, s) \mapsto t s$.

Write $v_{\alpha^{\prime}}=d t \wedge v^{\prime}+v^{\prime \prime}$ in the sense of (1.4.1). Then $v_{\alpha^{\prime \prime}}=d(s t) \wedge v^{\prime}(s t)$ $+v^{\prime \prime}(s t)=d t \wedge s v^{\prime}(s t)+\left(d s \wedge t v^{\prime}(s t)+v^{\prime \prime}(s t)\right)$.

Hence

$$
\left(F^{*} \int^{\prime} v\right)_{1_{I} \times \alpha}=\left(\int^{\prime} v\right)_{\alpha^{\prime}}=\int_{0}^{1} s v^{\prime}(s t) d t=\int_{0}^{s} v^{\prime}(t) d t .
$$

COROLLARY. Under the hypothesis of the lemma, $\int^{\prime} \int^{\prime}=0$.

For any element $u$ in a graded vector space, write $J u=(-1)^{\operatorname{deg} u} u$.

Take note that, for any form $w$ on $M, \int^{\prime} p_{0}^{*} w=0$ because the composite map $I \times P(M) \stackrel{F}{\rightarrow} P(M) \stackrel{p_{0}}{\rightarrow} M$ is given by $(t, \gamma) \mapsto \eta_{\gamma(0)}$ and is independent of $t$.

Definition 1.5.1. For forms $w_{1}, \ldots, w_{r}$ on $M$, define $\int w_{1}=\int^{\prime} p_{1}^{*} w_{1}$ and, for $r>1$,

$$
\int w_{1} \cdots w_{r}=\int^{\prime}\left(J_{1} \int w_{1} \cdots w_{r-1}\right) \wedge p_{1}^{*} w_{r} .
$$

Set $\int w_{1} \cdots w_{r}=1$ when $r=0$ and $=0$ when $r<0$.

Observe that $\int w_{1} \cdots w_{r}$ is of degree $\Sigma_{1 \leqslant i \leqslant r}\left(-1+\operatorname{deg} w_{i}\right)$ on $P(M)$. Since $\int w_{1} \cdots w_{r}=0$ if $\operatorname{deg} w_{i}=0$ for some $i, 1 \leqslant i \leqslant r$, it will be understood that each $w_{i}$ is of positive degree.

Elements of the graded subspace of $\Lambda(P(M))$ spanned by all $\int w_{1} \cdots w_{r}$, $r \geqslant 0$, will be called iterated integrals.

Proposition 1.5.2. For forms $w_{1}, \ldots, w_{r}$ on $M$,

$$
\begin{gathered}
d \int w_{1} \cdots w_{r}=\sum_{1 \leqslant i \leqslant r}(-1)^{i} \int J w_{1} \cdots J w_{i-1} d w_{i} w_{i+1} \cdots w_{r} \\
-\sum_{1 \leqslant i<r}(-1)^{i} \int J w_{1} \cdots J w_{i-1}\left(J w_{i} \wedge w_{i+1}\right) w_{i+2} \cdots w_{r} \\
\quad-p_{0}^{*} w_{1} \wedge \int w_{2} \cdots w_{r}+\left(J \int w_{1} \cdots w_{r-1}\right) \wedge p_{1}^{*} w_{r} .
\end{gathered}
$$


PROOF. The formula holds when $r=0$. For $r>0$,

$$
\begin{aligned}
& d \int w_{1} \cdots w_{r}= d \int^{\prime}\left(J \int w_{1} \cdots w_{r-1}\right) \wedge p_{1}^{*} w_{r} \\
& \text { according to Lemma 1.4.1, } \\
&=-\int^{\prime} d\left(\left(J \int w_{1} \cdots w_{r-1}\right) \wedge p_{1}^{*} w_{r}\right)+\left(J \int w_{1} \cdots w_{r-1}\right) \wedge p_{1}^{*} w_{r} \\
&=\int^{\prime}\left(\left(J d \int w_{1} \cdots w_{r-1}\right) \wedge p_{1}^{*} w_{r}\right) \\
&+(-1)^{r} \int J w_{1} \cdots J w_{r-1}\left(d w_{r}\right)+\left(J \int w_{1} \cdots w_{r-1}\right) \wedge p_{1}^{*} w_{r} .
\end{aligned}
$$

Hence the lemma follows by induction.

In the cases of $r=1$ and 2, we have formulas

$$
\begin{aligned}
d \int w= & -\int d w-p_{0}^{*} w+p_{1}^{*} w \\
d \int w_{1} w_{2}= & -\int d w_{1} w_{2}+\int J w_{1} d w_{2}+\int J w_{1} \wedge w_{2} \\
& -p_{0}^{*} w_{1} \wedge \int w_{2}+\left(J \int w_{1}\right) \wedge p_{1}^{*} w_{2} .
\end{aligned}
$$

In $\$ 1.4$ [24], a constructive definition of $\int w_{1} \cdots w_{r}$ was given. We are going to verify that the present definition agrees with that definition.

Write $\left.w_{i}^{\prime}=(\partial / \partial t)\right\lrcorner F^{*} p_{1}^{*} w_{i}$ and $u_{r}=F^{*} \int w_{1} \cdots w_{r}$, both of which are $\Lambda(P(M))$-valued functions on $I$. Since $F$ is a homotopy from $\eta \circ p_{0}$ to $1_{P(M)}$, we have

$$
u_{r}(1)=\int w_{1} \cdots w_{r} .
$$

If $\alpha: U \rightarrow P(M)$ is a plot, then

$$
\left.\left.\left(w_{i}^{\prime}\right)_{1, \times \alpha}=\frac{\partial}{\partial t}\right\lrcorner\left(F^{*} p_{1}^{*} w_{i}\right)_{1, \times \alpha}=\frac{\partial}{\partial t}\right\lrcorner\left(w_{i}\right)_{\phi_{\alpha}}
$$

which is $w_{i}(\alpha, \stackrel{\alpha}{\alpha})$ as defined in [24]. Therefore, in order that the two definitions agree, it suffices to verify that

$$
u_{r}(s)=\int_{0}^{s}\left(\int_{0}^{t_{r}}\left(\cdots \int_{0}^{t_{2}} w_{1}^{\prime}\left(t_{1}\right) d t_{1} \cdots\right) \wedge w_{r-1}^{\prime}\left(t_{r-1}\right) d t_{r-1}\right) \wedge w_{r}^{\prime}\left(t_{r}\right) d t_{r} .
$$

For $r>1, u_{r}$ is a $\Lambda(P(M))$-valued function on $I$ such that

$$
u_{r}(s)=\int_{0}^{s} v^{\prime}(t) d t
$$

where

$$
\left.\left.v^{\prime}(t)=\frac{\partial}{\partial t}\right\lrcorner F^{*}\left(J \int w_{1} \cdots w_{r-1} \wedge p_{1}^{*} w_{r}\right)=\frac{\partial}{\partial t}\right\lrcorner\left(J u_{r-1}(t) \wedge F^{*} p_{1}^{*} w_{r}\right) .
$$

According to Lemma 1.5.1, $u_{r-1}$ is also a $\Lambda(P(M))$-valued function on $I$ so 
ITERATED PATH INTEGRALS

841

that

$$
\left.v^{\prime}(t)=u_{r-1}(t) \wedge\left(\frac{\partial}{\partial t}\right\lrcorner F^{*} p_{1}^{*} w_{r}\right)=u_{r-1}(t) \wedge w_{r}^{\prime}(t)
$$

Finally, (1.5.4) follows by induction.

EXAMPLE 1.5.1. Let $w$ be a $p$-form on $M$ and let $\alpha: U \rightarrow P(M)$ be a compact plot with $\operatorname{dim} U=p-1>0$. Write

$$
w_{\phi_{\alpha}}=a(t, \xi) d t \wedge d \xi^{1} \wedge \cdots \wedge d \xi^{p-1}
$$

so that

$$
\left.\left.\left(\frac{\partial}{\partial t}\right\lrcorner F^{*} w\right)_{1, \times \alpha}=\frac{\partial}{\partial t}\right\lrcorner w_{\phi_{\alpha}}=a(t, \xi) d \xi^{1} \wedge \cdots \wedge d \xi^{p-1}
$$

and

$$
\left\langle\int w, \alpha\right\rangle=\int_{U}\left(\int_{0}^{1} a(t, \xi) d t\right) d \xi^{1} \wedge \cdots \wedge d \xi^{p-1}
$$

Therefore

$$
\left\langle\int w, \alpha\right\rangle=\int_{\phi_{\alpha}} w
$$

EXAMPLE 1.5.2. A closed Riemann surface $M$ of genus $p$ can be represented by a disc $D$ whose boundary is represented by a product of loops at $x_{0} \in M$ :

$$
\gamma=\alpha_{1} \beta_{1} \alpha_{1}^{-1} \beta_{1}^{-1} \cdots \alpha_{p} \beta_{p} \alpha_{p}^{-1} \beta_{p}^{-1} \text {. }
$$

Construct a plot $\sigma: I \rightarrow P(M)$ with $\sigma(0)=\eta_{x_{0}}$ and $\sigma(1)=\gamma$ such that the suspension map $\phi_{\alpha}$ maps the interior of $I \times I$ homeomorphically onto the interior of $D$.

Let $w_{1}$ and $w_{2}$ be closed 1-forms on $M$. Then

$$
\left\langle\int w_{1} w_{2}, \partial \sigma\right\rangle=\left\langle d \int w_{1} w_{2}, \sigma\right\rangle
$$

i.e.

$$
\left\langle\int w_{1} w_{2}, \partial \sigma\right\rangle=\left\langle-\int w_{1} \wedge w_{2}-\left(p_{0}^{*} w_{1}\right) \wedge \int w_{2}+\int w_{1} \wedge p_{1}^{*} w_{2}, \sigma\right\rangle .
$$

Since $p_{0} \sigma$ and $p_{1} \sigma$ are constant plots, $\left(p_{0}^{*} w_{1}\right)_{\sigma}=\left(p_{1}^{*} w_{2}\right)_{\sigma}=0$ and the r.h.s. of the above equation becomes

$$
\left\langle-\int w_{1} \wedge w_{2}, \sigma\right\rangle=-\left\langle w_{1} \wedge w_{2}, \phi_{\sigma}\right\rangle=-\int_{M} w_{1} \wedge w_{2} .
$$

On the other hand, $\partial \sigma=(\gamma)-\left(\eta_{x_{0}}\right)$, where $(\gamma)$ denotes the 0 -simplex (or 0-cube) of $P(M)$ at $\gamma$. Use formulas in [21] to compute the 1.h.s. of the equation, which becomes

$$
\begin{aligned}
\left\langle\int w_{1} w_{2},(\gamma)\right\rangle & =\sum\left\langle\int w_{1} w_{2},\left(\alpha_{i} \beta_{i} \alpha_{i}^{-1} \beta_{i}^{-1}\right)\right\rangle \\
& =\sum\left(\int_{\alpha_{i}} w_{1} \int_{\beta_{i}} w_{2}-\int_{\alpha_{i}} w_{2} \int_{\beta_{i}} w_{1}\right) .
\end{aligned}
$$


We obtain the identity

$$
\int_{M} w_{1} \wedge w_{2}=-\sum_{1 \leqslant i \leqslant p}\left(\int_{\alpha_{i}} w_{1} \int_{\beta_{i}} w_{2}-\int_{\alpha_{i}} w_{2} \int_{\beta_{i}} w_{1}\right),
$$

which is essentially the Riemann bilinear relation for $M$. (See [4].)

Definition 1.5.2. Let $\alpha, \beta: U \rightarrow P(M)$ be plots. Let $\tau: I \times U \rightarrow I$ be a $C^{\infty}$ map with $\tau(0, \xi)=0$ and $\tau(1, \xi)=1 \forall \xi \in U$. Then $\beta$ is a reparametrization of $\alpha$ via $\tau$ if

$$
\beta(\xi)(t)=\alpha(\xi)(\tau(t, \xi)), \quad \forall(t, \xi) \in I \times U .
$$

Proposition 1.5.3. If $\beta$ is a reparametrization of $\alpha$, then

$$
\left(\int w_{1} \cdots w_{r}\right)_{\beta}=\left(\int w_{1} \cdots w_{r}\right)_{\alpha}
$$

for any forms $w_{1}, \ldots, w_{r}$ on $M, r \geqslant 0$.

Proof. Let $\tilde{\tau}: I \times U \rightarrow I \times U$ be given by $(t, \xi) \mapsto(\tau(t, \xi), \xi)$. Write a form $\boldsymbol{v}$ on $I \times U$ as $v=d t \wedge v^{\prime}+v^{\prime \prime}$ with $\left.v^{\prime}=(\partial / \partial t)\right\lrcorner v$. Then

$$
\tilde{\tau}^{*} v=(\partial \tau(t, \xi) / \partial t) d t \wedge \tilde{\tau}^{*} v^{\prime}+\tilde{\tau}^{*} v^{\prime \prime}
$$

where $\tilde{\tau}^{*} v$ and $\tilde{\tau}^{*} v^{\prime \prime}$ are also $\Lambda(U)$-valued functions on $I$. If $w$ is a form on $M$, then

$$
\left(F^{*} p_{1}^{*} w\right)_{1, \times \beta}=(\partial \tau(t, \xi) / \partial t) \tilde{\tau}^{*}\left(F^{*} p_{1}^{*} w\right)_{1, \times \alpha}
$$

By using (1.5.4) together with (1.5.5) and observing that the iterated integral (1.1.1) does not depend on the parametrization of the interval $[a, b]$, we conclude the proof of this proposition.

REMARK. This proposition will also hold when Definition 1.5.2 is weakened by allowing $\tau(t, \xi)$ to be piecewise $C^{\infty}$ with respect to the variable $t$.

The following formula for the exterior multiplication of iterated integrals can be found in $\$ 4.1[24]$ :

$$
\int w_{1} \cdots w_{r} \wedge \int w_{r+1} \cdots w_{r+s}=\sum \varepsilon_{\sigma} \int w_{\sigma(1)} \cdots w_{\sigma(r+s)}
$$

summing over those permutations $\sigma$ of $r+s$ letters with

$$
\sigma^{-1}(1)<\cdots<\sigma^{-1}(r), \quad \sigma^{-1}(r+1)<\cdots<\sigma^{-1}(r+s),
$$

where $\varepsilon_{\sigma}= \pm 1$ depends on $\sigma$ and also deg $w_{i}, 1 \leqslant i \leqslant r+s$.

1.6. Products of path space plots. We are going to define two related partial multiplications of plots of $P(M)$, which will assume an essential role in uncovering the geometrical significance of iterated integrals.

Definition 1.6.1. Let $x_{0}, x_{1} \in M$. A plot $\alpha: U \rightarrow P(M)$ is said to be from $x_{0}\left(\right.$ resp. to $\left.x_{1}\right)$ if $\alpha(\xi)(0)=x_{0}$ (resp. $\left.\alpha(\xi)(1)=x_{1}\right) \forall \xi \in U$.

Let $\alpha, \beta: U \rightarrow P(M)$ be plots with $\alpha(\xi)(1)=\beta(\xi)(0) \forall \xi \in U$. Define the set map

$\alpha \beta: U \rightarrow$ the set of piecewise smooth paths in $M$

such that $\alpha \beta(\xi)(t)=\alpha(\xi)(2 t)$ for $0 \leqslant t \leqslant \frac{1}{2}$ and $=\beta(\xi)(2 t-1)$ for $\frac{1}{2} \leqslant t \leqslant$ 1. 
Define the plot $\alpha^{-1}: U \rightarrow P(M)$ such that $\alpha^{-1}(\xi)(t)=\alpha(\xi)(1-t)$.

If $\alpha: U \rightarrow P(M)$ is a plot to $x$ and if $\alpha^{\prime}: U^{\prime} \rightarrow P(M)$ is a plot from the same point $x$, define the set map

$\alpha \times \alpha^{\prime}: U \times U^{\prime} \rightarrow$ the set of piecewise smooth paths in $M$

such that $\left(\alpha \times \alpha^{\prime}\right)\left(\xi, \xi^{\prime}\right)(t)=\alpha(\xi)(2 t)$ for $0 \leqslant t \leqslant \frac{1}{2}$ and $=\alpha^{\prime}\left(\xi^{\prime}\right)(2 t-1)$ for $\frac{1}{2} \leqslant t \leqslant 1$.

We shall say that the product plot $\alpha \beta$ (resp. $\alpha \times \alpha^{\prime}$ ) is well defined, if the map $\alpha \beta$ (resp. $\alpha \times \alpha^{\prime}$ ) is well defined and, furthermore, is a plot of $P(M)$.

When the product plots $\alpha \beta$ and $\alpha \times \alpha^{\prime}$ are well defined, the following formulas can be verified as in $\S 1$ [24].

$$
\begin{aligned}
& \text { (1.6.1) }\left(\int w_{1} \cdots w_{r}\right)_{\alpha \beta}=\sum_{0 \leqslant i \leqslant r}\left(\int w_{1} \cdots w_{i}\right)_{\alpha} \wedge\left(\int w_{i+1} \cdots w_{r}\right)_{\beta}, \\
& \text { (1.6.2) }\left(\int w_{1} \cdots w_{r}\right)_{\alpha \times \alpha^{\prime}}=\sum_{0 \leqslant i \leqslant r}\left(\int w_{1} \cdots w_{i}\right)_{\alpha} \times\left(\int w_{i+1} \cdots w_{r}\right)_{\alpha^{\prime}}, \\
& \text { (1.6.3) }\left\langle\int w_{1} \cdots w_{r}, \alpha \times \alpha^{\prime}\right\rangle=\sum_{0 \leqslant i \leqslant r}\left\langle\int w_{1} \cdots w_{i}, \alpha\right\rangle\left\langle\int w_{i+1} \cdots w_{r}, \alpha^{\prime}\right\rangle,
\end{aligned}
$$

provided both $\alpha$ and $\alpha^{\prime}$ are compact plots.

$$
\left(\int w_{1} \cdots w_{r}\right)_{\alpha \alpha^{-1}}=0, \quad r \geqslant 1 .
$$

Recall that a plot chain of $P(M)$ on $U$ is a finite formal sum of the type $c=\sum n_{i} \alpha_{i}$ where $n_{i} \in Z$ and each $\alpha_{i}: U \rightarrow P(M)$ is a plot. We say that the plot chain $c$ is reduced if $\sum n_{i}=0$.

Definition 1.6.2. A plot chain $c$ of $P(M)$ on a compact convex set is of order $s$ if, for any forms $w_{1}, \ldots, w_{r}$ on $M,\left\langle\int w_{1} \ldots w_{r}, c\right\rangle=0$ provided $r<s$.

Definition 1.6.3. A plot chain $c$ of $P(M)$ on $U$ is strongly of order $s$ if, for any forms $w_{1}, \ldots, w_{r}$ on $M,\left(\int w_{1} \ldots w_{r}\right)_{c}=0$ provided $r<s$.

For example, any compact plot $\alpha: U \rightarrow P(M)$ with $\operatorname{dim} U>0$ is of order 1 , because $\langle 1, \alpha\rangle=0$. On the other hand, $\alpha$ is not strongly of order 1 , for $1_{\alpha}=1 \neq 0$. Every reduced plot chain of $P(M)$ is strongly of order 1 .

Let $c=\sum n_{i} \alpha_{i}$ and $c^{\prime}=\sum n_{j}^{\prime} \alpha_{j}^{\prime}$ be plot chains of $P(M)$ on $U$ and $U^{\prime}$ respectively. We say that the product plot chain

$$
c \times c^{\prime}=\sum_{i, j} n_{i} n_{j}^{\prime} \alpha_{i} \times \alpha_{j}^{\prime}
$$

is well defined if each product plot $\alpha_{i} \times \alpha_{j}^{\prime}$ with $n_{i} n_{j}^{\prime} \neq 0$ is well defined. Similarly, we say that the product chain

$$
c c^{\prime}=\sum_{i, j} n_{i} n_{j}^{\prime} \alpha_{i} \alpha_{j}^{\prime}
$$

is well defined if each product plot $\alpha_{i} \alpha_{j}^{\prime}$ with $n_{i} n_{j}^{\prime} \neq 0$ is well defined. The following formulas are direct consequences of (1.6.1) and (1.6.3): 


$$
\left(\int w_{1} \cdots w_{r}\right)_{c c^{\prime}}=\sum_{0 \leqslant i \leqslant r}\left(\int w_{1} \cdots w_{i}\right)_{c} \wedge\left(\int w_{i+1} \cdots w_{r}\right)_{c^{\prime}}
$$

$$
\left\langle\int w_{1} \cdots w_{r}, c \times c^{\prime}\right\rangle=\sum_{0<i<r}\left\langle\int w_{1} \cdots w_{i}, c\right\rangle\left\langle\int w_{i+1} \cdots w_{r}, c^{\prime}\right\rangle
$$

provided $U$ and $U^{\prime}$ are compact.

The above formulas lead to the next assertion.

Lemma 1.6.1. Let $c$ and $c^{\prime}$ be plot chains of $P(M)$ such that the product plot chain $c \times c^{\prime}$ (resp. $\left.c c^{\prime}\right)$ is well defined. If $c$ is of order $r$ (resp. strongly of order $r$ ) and if $c^{\prime}$ is of order $s$ (resp. strongly of order s), then $c \times c^{\prime}$ (resp. $\left.c c^{\prime}\right)$ is of order $r+s$ (resp. strongly of order $r+s)$.

COROLlary. If $c_{1}, \ldots, c_{s}$ are plot chains of $P(M)$ of order 1 (resp. strongly of order 1) and if the product plot chain $c_{1} \times \ldots \times c_{s}\left(\right.$ resp. $\left.c_{1} \ldots c_{s}\right)$ is well defined, then it is of order $s$ (resp. strongly of order s).

REMARK. We have not indicated a grouping for the multiplication in the product plot chain $c_{1} \times \ldots \times c_{s}$ (resp. $c_{1} \ldots c_{s}$ ) for such a grouping will be immaterial for our subsequent discussions owing to Proposition 1.5.2 and the pertinent remark.

\section{CHAPTER II. LOOP SPACE COHOMOLOGY}

Let $M$ be a differentiable space, and let $x_{0} \in M$. We associate to every differential graded subalgebra $A$ of $\Lambda(M)$ a differential graded subalgebra $A^{\prime}$ of $\Lambda(P(M))$. Denote by $A_{x_{0}}^{\prime}$ the restriction of $A^{\prime}$ on the smooth loop space $\Omega M$ at $x_{0}$. Then $A_{x_{0}}^{\prime}$ is a subcomplex of $\Lambda(\Omega M)$ spanned by iterated integrals. The main objective of this chapter is to determine the cohomology $H\left(A_{x_{0}}^{\prime}\right)$. Theorem 2.3.1 asserts that, under reasonable conditions, $H\left(A_{x_{0}}^{\prime}\right)$ is the real loop space cohomology of $M$ for the simply connected case. Theorem 2.6.1 characterizes $H^{0}\left(A_{x_{0}}^{\prime}\right)$ in the nonsimply connected case. (In Sullivan's theory of minimal models, there are results of similar nature. See [66] and $\$ 3$ [31].) Recently, in analyzing maps from the bar construction of $\Lambda(M)$ to $\Lambda(P(M))$, Gugenheim [75] gives a proof of a more general version of Theorem 4.7.1 [24].

Most material in this chapter can be found, in essence, in [24] and [26].

2.1. Differential graded algebras of iterated integrals. Let $A$ be a differential graded subalgebra of $\Lambda(M)$. Denote by $A^{\prime}$ the subcomplex of $\Lambda(P(M))$ spanned by

$$
p_{0}^{*} w^{\prime} \wedge \int w_{1} \cdots w_{r} \wedge p_{1}^{*} w^{\prime \prime}, \quad w^{\prime}, w^{\prime \prime}, w_{1}, \ldots, w_{r} \in A, r \geqslant 0 .
$$

It follows from (1.5.6) that $A^{\prime}$ is closed under the exterior multiplication. Indeed, $A^{\prime}$ is the smallest differential graded subalgebra of $\Lambda(P(M))$ that contains both $p_{0}^{*} A$ and $p_{1}^{*} A$ and is stable with respect to the Poincare operator $\int^{\prime}$.

The two maps $A \rightarrow A^{\prime}$ respectively given by $w \mapsto p_{0}^{*} w$ and $p_{1}^{*} w$ induce the same cohomology homomorphism $H(A) \rightarrow H\left(A^{\prime}\right)$ because they are chain homotopic via a chain homotopy given by $w \mapsto \int w$. 
THEOREM 2.1.1. The differential graded map $A \rightarrow A^{\prime}$ given by $w$ $p_{0}^{*} w$ is a chain equivalence.

PRoof. If $\eta: M \rightarrow P(M)$ is the constant path map, consider the differential graded map $A^{\prime} \rightarrow A$ given by $u \mapsto \eta^{*} u$. Since $\eta^{*} p_{0}^{*} w=w$, it remains to show that the differential graded map $A^{\prime} \rightarrow A^{\prime}$ given by $u \mapsto p_{0}^{*} \eta^{*} u$ is chain homotopic to $1_{A^{\prime}}$. Hence this theorem is a consequence of Lemma 1.4.1 and the definition of the Poincare operator $\int^{\prime}$.

For $x_{0}, x_{1} \in M$, denote by $P\left(M ; x_{0}, x_{1}\right)$ the differentiable subspace of $P(M)$ consisting of all paths from $x_{0}$ to $x_{1}$. If $\alpha$ is a plot of $P\left(M ; x_{0}, x_{1}\right)$, then both $p_{0} \circ \alpha$ and $p_{1} \circ \alpha$ are constant maps so that $p_{0}^{*} w=p_{1}^{*} w=0$ for any form $w$ of positive degree on $M$.

Denote by $\int_{x_{0}}^{x_{1}} w_{1} \cdots w_{r}$ the restriction of $\int w_{1} \cdots w_{r}$ on $P\left(M ; x_{0}, x_{1}\right)$. The exterior differentiation formula becomes

$$
\begin{aligned}
& d \int_{x_{0}}^{x_{1}} w_{1} \cdots w_{r}=\sum_{1 \leqslant i \leqslant r}(-1)^{i} \int_{x_{0}}^{x_{1}} J w_{1} \cdots J w_{i-1} d w_{i} w_{i+1} \cdots w_{r} \\
& -\sum_{1 \leqslant i<r}(-1)^{i} \int_{x_{0}}^{x_{1}} J w_{1} \cdots J w_{i-1}\left(J w_{i} \wedge w_{i+1}\right) w_{i+2} \cdots w_{r} .
\end{aligned}
$$

If $A$ is a differential graded subalgebra of $\Lambda(M)$, denote by $A_{x_{0}, x_{1}}^{\prime}$ the restriction of $A^{\prime}$ on $P\left(M ; x_{0}, x_{1}\right)$. Then $A_{x_{0}, x_{1}}^{\prime}$ is spanned by all $\int_{x_{0}}^{x_{1}} w_{1} \cdots \cdots w_{r}$ with $w_{1}, \ldots, w_{r} \in A, r \geqslant 0$.

Write $\Omega M=P\left(M ; x_{0}, x_{0}\right)$, which is the loop space of $M$ at a given base point $x_{0}$. Set $A_{x_{0}}^{\prime}=A_{x_{0}, x_{0}}^{\prime}$.

\subsection{The smoothing property.}

Definition 2.2.1. A map $\alpha: U \rightarrow P(M)$ is said to be in equilibrium about $\left(t_{0}, \xi_{0}\right)$ if, for $(t, \xi)$ in a neighborhood of $\left(t_{0}, \xi_{0}\right)$ in $I \times U, \alpha(\xi)(t)=\alpha(\xi)\left(t_{0}\right)$. A plot $\alpha: U \rightarrow P(M)$ is said to end (resp., to start) smoothly if it is in equilibrium about $(1, \xi)(\operatorname{resp} .(0, \xi)), \forall \xi \in U$.

Lemma 2.2.1. Every differentiable space $M$ satisfies the following property (which will be called the smoothing property): For every continuous function a: $U \rightarrow I$ on a convex set $U$, if a map $\alpha: U \rightarrow P(M)$ is in equilibrium about $(a(\xi), \xi), \forall \xi \in U$ and if, for the suspension map $\phi_{\alpha}$, the restrictions

$$
\phi_{\alpha} \mid\{(t, \xi) \in I \times U: 0 \leqslant t \leqslant a(\xi)\}
$$

and

$$
\phi_{\alpha} \mid\{(t, \xi) \in I \times U: a(\xi) \leqslant t \leqslant 1\}
$$

are both differentiable maps, then $\alpha$ is a plot of $P(M)$.

Proof. The restriction of $\phi_{\alpha}$ to a sufficiently small convex neighborhood of any point in $I \times U$ is a plot of $M$. Hence, according to the condition (d) of Definition 1.1.1, $\phi_{\alpha}$ is a plot of $M$.

Corollary. Let $\alpha, \beta: U \rightarrow P(M)$ be plots with $\alpha(\xi)(1)=\beta(\xi)(0), \forall \xi \in U$. Let $a: U \rightarrow(0,1)$ be a $C^{\infty}$ function. If $\alpha$ ends smoothly and if $\beta$ starts smoothly, then the map $\mu: U \rightarrow P(M)$ given by 


$$
\mu(\xi)(t)=\left\{\begin{array}{l}
\alpha(\xi)(t / a(\xi)), \quad 0 \leqslant t \leqslant a(\xi), \\
\beta(\xi)([t-a(\xi)] /[1-a(\xi)]), \quad a(\xi) \leqslant t \leqslant 1,
\end{array}\right.
$$

is a plot of $P(M)$.

LEMMA 2.2.2. If $\alpha, \beta$ and $\mu$ are plots as given in the above corollary, then

$$
\left(\int w_{1} \cdots w_{r}\right)_{\mu}=\sum_{0 \leqslant i \leqslant r}\left(\int w_{1} \cdots w_{i}\right)_{\alpha} \wedge\left(\int w_{i+1} \cdots w_{r}\right)_{\beta}
$$

for any forms $w_{1}, \ldots, w_{r}$ on $M$.

Proof. Let $\tau: I \times U \rightarrow I \times U$ be the piecewise $C^{\infty}$ map such that, $\forall \xi \in U, \tau$ maps $[0,1 / 2] \times\{\xi\}$ and $[1 / 2,1] \times\{\xi\}$ linearly onto $[0, a(\xi)] \times\{\xi\}$ and $[a(\xi), 1] \times\{\xi\}$ respectively. Then the product plot $\alpha \beta$, as defined in $\S 1.6$, is a reparametrization of $\mu$. Hence the lemma follows from Proposition 1.5.2 and (1.6.1).

Corollary. Let $\alpha: U \rightarrow P(M)$ be a plot ending smoothly to $x_{0}$ and $\alpha^{\prime}$ : $U^{\prime} \rightarrow P(M)$, a plot starting smoothly from $x_{0}$. If $\nu: U \times U^{\prime} \rightarrow P(M)$ is a plot given by

$$
\nu\left(\xi, \xi^{\prime}\right)(t)=\left\{\begin{array}{l}
\alpha(\xi)\left(t / a\left(\xi, \xi^{\prime}\right)\right), \quad 0 \leqslant t \leqslant a\left(\xi, \xi^{\prime}\right) \\
\alpha^{\prime}\left(\xi^{\prime}\right)\left(\left[t-a\left(\xi, \xi^{\prime}\right)\right] /\left[1-a\left(\xi, \xi^{\prime}\right)\right]\right), \quad a\left(\xi, \xi^{\prime}\right) \leqslant t \leqslant 1,
\end{array}\right.
$$

where a: $U \times U^{\prime} \rightarrow(0,1)$ is a $C^{\infty}$ function, then

$$
\left(\int w_{1} \cdots w_{r}\right)_{\nu}=\sum_{0 \leqslant i \leqslant r}\left(\int w_{1} \cdots w_{i}\right)_{\alpha} \times\left(\int w_{i+1} \cdots w_{r}\right)_{\alpha^{\prime}} .
$$

LEMmA 2.2.3. Let $x_{0}, x_{1} \in M$. Given any forms $w_{i}$ and simplices $\sigma_{i}$ of $M$ with $\operatorname{deg} w_{i}=\operatorname{deg} \sigma_{i}=n_{i}>0,1 \leqslant i \leqslant s$, there exists a compact plot chain $c$ of $P\left(M ; x_{0}, x_{1}\right)$ strongly of order $s$ such that

$$
\left\langle\int w_{1} \cdots w_{s}, c\right\rangle=\left(\int_{\sigma_{1}} w_{1}\right) \cdots\left(\int_{\sigma_{s}} w_{s}\right) .
$$

Proof. Let $\hat{\sigma}_{i}$ be the $\left(n_{i}-1\right)$-cube of $P(M)$ from $x_{i}^{\prime}$ to $x_{i}^{\prime \prime}$ as defined in $\S \mathrm{A} 2$. Let $\gamma_{i}^{\prime}$ (resp. $\gamma_{i}^{\prime}$, and $\gamma$ ) be a 0 -cube of $P(M)$ from $x_{0}$ to $x_{i}^{\prime}$ (resp. from $x_{i}^{\prime \prime}$ to $x_{0}$ and from $x_{0}$ to $x_{1}$ ), which starts and ends smoothly. Let

$$
\varepsilon_{i}: I^{n_{i}-1} \rightarrow P(M)
$$

be the constant map whose image is the constant loop $\eta_{x_{0}}$. Then $c_{i}=\gamma_{i}^{\prime} \times \hat{\sigma}_{i}$ $\times \gamma_{i}^{\prime \prime}-\varepsilon_{i}$ is a plot chain on $I^{n_{i}-1}$ which is strongly of order 1 , and $\left\langle\int w_{i}, c_{i}\right\rangle=\int_{\sigma_{i}} w_{i}$. Set $c=c_{1} \times \cdots \times c_{s} \times \gamma$, which is a compact plot chain strongly of order $s$. Hence, according to (1.6.6),

$$
\begin{aligned}
\left\langle\int w_{1} \cdots w_{s}, c\right\rangle & =\left\langle\int w_{1} \cdots w_{s}, c_{1} \times \cdots \times c_{s}\right\rangle\langle 1, \gamma\rangle \\
& =\left\langle\int w_{1}, c_{1}\right\rangle \cdots\left\langle\int w_{s}, c_{s}\right\rangle .
\end{aligned}
$$


PROPOSITION 2.2.4. The canonical homomorphism $H_{D R}(M) \rightarrow H^{*}(M ; k)$ via integration preserves the cup product.

Proof. Let $w^{\prime}$ and $w^{\prime \prime}$ be closed $p$ - and $q$-forms on $M, p, q>0$. Let $\sigma$ : $\Delta^{n} \rightarrow M$ be a simplex, where $n=p+q$. Then

$$
\int_{\sigma} w^{\prime} \wedge w^{\prime \prime}=\left\langle\int w^{\prime} \wedge w^{\prime \prime}, \hat{\sigma}\right\rangle=(-1)^{p}\left\langle d \int w^{\prime} w^{\prime \prime}, \hat{\sigma}\right\rangle
$$

by (A2.1) and Lemma 2.2.2,

$$
=(-1)^{p}\left\langle\int w^{\prime} w^{\prime \prime}, \widehat{\partial \sigma}\right\rangle-\int_{(p)^{n^{\prime}}} w^{\prime} \int_{\sigma_{(4)}} w^{\prime \prime} .
$$

Hence, if $z$ is an $n$-cycle in $\Delta(M)$, then

$$
\int_{z} w^{\prime} \wedge w^{\prime \prime}=-\left\langle w^{\prime} \otimes w^{\prime \prime}, \Delta z\right\rangle
$$

where $\Delta$ denotes the Alexander-Whitney diagonal, and the negative sign is due to our sign convention for the formula (A2.1).

2.3. A theorem on loop space cohomology. Recall that $k$ is the field of real (or complex) numbers.

THEOREM 2.3.1. Let $M$ be a topological differentiable space with a base point $x_{0}$ and having the following properties:

(a) The underlying topological space ${ }_{T} M$ is simply connected with homology of finite type.

(b) The inclusion $\Delta(M)_{x_{0}} \subset \Delta\left({ }_{T} M\right)$ induces an isomorphism $H\left(\Delta(M)_{x_{0}}\right) \approx$ $H_{*}\left(T_{T} M\right)$.

If $A$ is a differential graded subalgebra of $د(M)$ such that $H(A) \approx H^{*}\left({ }_{T} M ; k\right)$ via integration over $\Delta(M)_{x_{0}}$, then there is an isomorphism

$$
H\left(A_{x_{0}}^{\prime}\right) \approx H^{*}\left(\Omega_{T} M ; k\right) .
$$

REMARK 1. The above theorem has been proved in [24]. In his work [75], Gugenheim gives a simpler proof without using the cobar construction. The proof as given in $\$ 2.5$ will enable us to include in the theorem the following additional assertion: "If the inclusion induces an isomorphism

$$
H^{*}\left(\Omega_{T} M ; k\right) \approx H^{*}(\Omega M ; k)
$$

then (2.3.1) is an isomorphism of graded algebra via integration."

RemarK 2. Every simply connected mainfold $M$ with homology of finite type meets the conditions of the theorem including the isomorphism (2.3.2).

ExAMPLE 2.3.1. Let $w$ be an $n$-form on $S^{n}, n>1$, so that $\iint_{s^{n}} w=1$. Let $A$ be the differential graded subalgebra of the de Rham complex $\Lambda\left(S^{n}\right)$ spanned by $1 \in \Lambda^{0}\left(S^{n}\right)$ and $w$. Then each $u_{r}=\int_{x_{0}}^{x_{0} w} \cdots w$ ( $r$ times) is a closed $r(n-1)$-form on $\Omega S^{n}$, where $x_{0} \in S^{n}$. Thus $H\left(A_{x_{0}}^{\prime}\right)=A_{x_{0}}^{\prime}$. It follows from the theorem that $A_{x_{0}}^{\prime} \approx H^{*}\left(\Omega_{T} S^{n}\right)$. Since $A_{x_{0}}^{\prime}$ has a basis consisting of $1, u_{1}$, $u_{2}, \ldots$, the $q$ th Betti number of the topological loop space of $S^{n}$ is equal to 1 when $q=r(n-1), r \geqslant 0$, and is equal to 0 otherwise. As a matter of fact, it will be shown in Example 3.1.1 that $u_{r}, r \geqslant 0$, represent integral de Rham cohomology classes. Consequently $J u_{r} \wedge u_{s}=n_{r s} u_{r+s}$, where $n_{r s}$ is an integer. 
In particular, when $n$ is odd, it can be seen through the formula (1.5.6) and its detailed description in §4.1 [24] that $n_{r s}=\left(\begin{array}{c}r+s \\ \mathrm{r}\end{array}\right)$. (The cohomology ring $H^{*}\left(\Omega S^{n}\right)$ was determined by Serre [58].)

Let $f: S^{2 n-1} \rightarrow S^{n}, n$ even, be a differentiable map such that $f\left(y_{0}\right)=x_{0}$. Then $(\Omega f)^{*} \int_{x_{0}}^{x_{0}} w w=\int_{y_{0}}^{y_{0}} f^{*} w f^{*} w$ represents an integral cohomology class. If $\alpha$ is a cycle representing a generator of $H_{2 n-2}\left(\Omega_{T} S^{2 n-1}\right)$, then $\left\langle\int_{y_{0}}^{y_{0}} f^{*} w f^{*} w, \alpha\right\rangle$ is an integer equal to the degree of the induced homomorphism $H_{2 n-2}\left(\Omega_{T} S^{2 n-1}\right) \rightarrow H_{2 n-2}\left(\Omega_{T} S^{n}\right)$ and is therefore the Hopf invariant of the $\operatorname{map} f[41]$.

In [70], J. H. C. Whitehead computed Hopf invariants by using a twice iterated integral. (See also [71].) Our version is essentially the same as his but offers a systematic approach.

2.4. Filtration and Massey products. We shall subsequently prove Theorem 2.3.1 by pairing a cohomological spectral sequence arising from a filtration of $A_{x_{0}}^{\prime}$ and homological spectral sequence of the cobar construction $F\left(\Delta(M)_{x_{0}}\right)$. Let us discuss now the filtration of $A_{x_{0}}^{\prime}$, which is itself geometrically meaningful.

Let $M$ be a differentiable space, and let $A$ be a differential graded subalgebra of $\Lambda(M)$. For $x_{0} \in M$, the complex $A_{x_{0}}^{\prime}$ has a descending filtration

$$
k=A_{x_{0}}^{\prime}(0) \subset \cdots \subset A_{x_{0}}^{\prime}(-s) \subset \cdots
$$

such that $A_{x_{0}}^{\prime}(-s)$ is the subcomplex spanned by all $\int_{x_{0}}^{x_{0}} w_{1} \cdots w_{r}, w_{1}, \ldots, w_{r}$ $\in A, 0 \leqslant r \leqslant s$. Set $A_{x_{0}}^{\prime}(s)=0$ for $s>0$.

There is a linear map

$$
A \rightarrow A_{x_{0}}^{\prime}(-1)
$$

given by $w \mapsto \int_{x_{0}}^{x_{0}} w^{2}$. By using the same argument as for Lemma 4.2.1 [24] and suitable reparametrization for smoothing plots, we can make the next assertion.

Proposition 2.4.1. Let $M$ be path connected, i.e. $H_{0}(M)=Z$. Then the kernel of the linear map (2.4.1) is $A^{0} \oplus A^{1} \cap d \Lambda^{0}(M)$.

In other words, if $w$ is a $p$-form on $M$, then $\int_{x_{0}}^{x_{0} w}$ determines $w$ uniquely when $p>1$ and up to the difference of an exact 1 -form when $p=1$.

Since $d \int_{x_{0}}^{x_{0}} w=-\int_{x_{0}}^{x_{0}} d w$, for any $p$-form $w, p>0$, on $M, w$ is closed if and only if $\int_{x_{0}}^{x_{0} w}$ is closed. If $u \in A_{x_{0}}^{\prime}(-s)$ and $d u \in A_{x_{0}}^{\prime}(-1)$, then there exists a unique $w \in A$ such that $d u=\int_{x_{0}}^{x_{0}} w$. Moreover $w$ is closed but not necessarily exact. Therefore $w$ represents a cohomology class. This is precisely the situation where Massey products [46], [51] arise.

For illustration, we describe how triple Massey products are obtained in our context: Let $w_{1}, w_{2}, w_{3}$ be closed forms in $A$, and let $w_{12}, w_{23} \in A$ satisfy the condition

Consider

$$
d w_{12}-J w_{1} \wedge w_{2}=0, \quad d w_{23}-J w_{2} \wedge w_{3}=0 .
$$

$$
u=\int_{x_{0}}^{x_{0}}\left(w_{1} w_{2} w_{3}+w_{1} w_{23}+w_{12} w_{3}\right) \in A_{x_{0}}^{\prime}(-3),
$$


whose differential is

$$
d u=\int_{x_{0}}^{x_{0}}\left(J w_{1} \wedge w_{23}+J w_{12} \wedge w_{3}\right) \in A_{x_{0}}^{\prime}(-1)
$$

The preimage of $d u$ under the map (2.4.1) is the closed form $J w_{1} \wedge w_{23}+$ $J w_{12} \wedge w_{3}$, whose cohomology class is called a Massey triple product.

If the above Massey product is trivial in $H(A)$, then

$$
d w_{123}-J w_{1} \wedge w_{23}-J w_{12} \wedge w_{3}=0
$$

for some $w_{123} \in A$. We obtain a closed form

$$
\int_{x_{0}}^{x_{0}}\left(w_{1} w_{2} w_{3}+w_{1} w_{23}+w_{12} w_{3}+w_{123}\right)
$$

in $A_{x_{0}}^{\prime}(-3)$, which represents a differentiable version of Kraines' cohomology class for loop spaces [47].

2.5. Pairing with the cobar construction. As in the Appendix, let $C=$ $\Delta(M)_{x_{0}}$. The cobar construction $F(C)$ has an ascending filtration $\left\{F_{s}(C)\right\}$ such that $F_{-s}(C)$ is spanned by all $\left[\sigma_{1}|\cdot \cdot| \sigma_{r}\right], r \geqslant s \geqslant 0$, and $F_{s}(C)=$ $F(C)$ for $s>0$. Denote by $\left\{E^{r}, d^{r}\right\}_{r \geqslant 0}$ the resulting spectral sequence. Then $E_{-s}^{0}=F_{-s}(C) / F_{-s-1}(C)$, and $E_{s}^{0}=0$ for $s>0$.

Let $C_{*-1}$ be the chain complex obtained from the reduced chain complex of $C$ through lowering the degree by 1 . That is $\left(C_{*-1}\right)_{q}=0$ for $q<1$, and $\left(C_{*-1}\right)_{q}=C_{q+1}, q \geqslant 0$. There are homomorphisms

$$
\otimes_{Z}^{s} C_{*-1} \rightarrow F_{-s}(C) / F_{-s-1}(C), \quad s \geqslant 1,
$$

given by $\sigma_{1} \otimes \cdots \otimes \sigma_{s} \mapsto\left[\sigma_{1}|\cdots| \sigma_{s}\right]+F_{-s-1}(C)$. Since

$d_{F}\left[\sigma_{1}|\cdots| \sigma_{s}\right] \equiv \sum(-1)^{i}\left[J \sigma_{1}|\cdots| J \sigma_{i-1}\left|\partial \sigma_{i}\right| \sigma_{i+1}|\cdots| \sigma_{r}\right] \bmod F_{-s-1}(C)$, we may verify that (2.5.1) is a chain map, which induces an isomorphism

$$
H\left(\otimes_{Z}^{s} C_{*-1}\right) \approx E_{-s}^{1}, \quad s \geqslant 0 .
$$

Note that $E_{0}^{1}=Z$ and $E_{s}^{1}=0$ when $s>0$.

The cochain complex $B=\operatorname{Hom}_{Z}(F(C), k)$ has a descending filtration

$$
k=B(0) \subset \cdots \subset B(-s) \subset \cdots
$$

where $B(-s)$ is the subcomplex of $B$ orthogonal to $F_{-s-1}(C)$, and, for $s>0$, $B(s)=0$. Let $\left\{E_{r}, d_{r}\right\}_{r} \geqslant 0$ be the resulting spectral sequence. If $H(C ; k)$ is of finite type, then (2.5.2) yields

$$
E_{1}^{-s} \approx \otimes^{s} H^{*}\left(C_{*-1} ; k\right), \quad s \geqslant 1 .
$$

Now the descending filtration $\left\{A_{x_{0}}^{\prime}(s)\right\}$ of $A_{x_{0}}^{\prime}$ also gives rise to a spectral sequence $\left\{\xi_{r}, b_{r}\right\}_{r \geqslant 0}$ with

$$
\mathbb{E}_{0}^{-s}=A_{x_{0}}^{\prime}(-s) / A_{x_{0}}^{\prime}(-s+1) \text {. }
$$

According to $\$ 4.3$ [24], there are cochain maps

$$
\bigotimes^{s}\left(A^{*-1}\right) \rightarrow A_{x_{0}}^{\prime}(-s) / A_{x_{0}}^{\prime}(-s+1), \quad s \geqslant 1,
$$

given by 


$$
w_{1} \otimes \cdots \otimes w_{s} \mapsto \int_{x_{0}}^{x_{0}} w_{1} \cdots w_{s}+A^{\prime}(-s+1),
$$

where the cochain complex $A^{*-1}$ is obtained from $A$ by setting $\left(A^{*-1}\right)^{p}=0$ when $p<0,=A^{1} / d A^{0}$ when $p=0$, and $=A^{p+1}$ when $p>0$.

Consider the pairing

$$
A_{x_{0}}^{\prime} \times F(C) \rightarrow k
$$

given by

$$
\left(\int_{x_{0}}^{x_{0}} w_{1} \cdots w_{r},\left[\sigma_{1}|\cdots| \sigma_{s}\right]\right) \mapsto\left\langle\int w_{1} \cdots w_{r}, \mu_{F}\left[\sigma_{1}|\cdots| \sigma_{s}\right]\right\rangle,
$$

where $\mu_{F}$ is the chain map given in $\S \mathrm{A} 2$. This pairing induces a cochain map

$$
A_{x_{0}}^{\prime} \rightarrow \operatorname{Hom}_{Z}(F(C), k) \text {. }
$$

In the remainder of this section, we are going to verify that the cochain map (2.5.5) preserves filtration and induces, under the hypotheses of Theorem 2.3.1 except the 1-connectedness condition for ${ }_{T} M$,

$$
\mathbb{E}_{r}^{-s} \approx E_{r}^{-s}
$$

for $r=1$ and therefore for any $r>1$.

LEMMA 2.5.1. If $\sigma_{1}, \ldots, \sigma_{s}$ are simplices of $C=\Delta(M)_{x_{0}}$, then the plot chain $\mu_{F}\left[\sigma_{1}|\cdots| \sigma_{s}\right]$ is of order $s$ (Definition 1.6.1).

Proof. Recall that each $\mu_{F}\left[\sigma_{i}\right]=\hat{\sigma}_{i}$ (or $\hat{\sigma}_{i}-\left(\eta_{x_{0}}\right)$ in case of $\operatorname{deg} \sigma_{i}=1$ ) is a plot chain of order 1 . For $s>1, \mu_{F}\left[\sigma_{1}|\cdots| \sigma_{s}\right]$ differs from the product $\mu_{F}\left[\sigma_{1}|\cdots| \sigma_{s-1}\right] \times \mu_{F}\left[\sigma_{s}\right]$ only by reparametrization of corresponding cubes in the sense of Definition 1.5.2. If $w_{1}, \ldots, w_{r}$ are forms on $M$, then Lemma 2.2.2 implies that

$$
\left(\int w_{1} \cdots w_{r}\right)_{\mu_{f}\left[\sigma_{1}|\cdots| \sigma_{1}\right]}=\left(\int w_{1} \cdots w_{r}\right)_{\mu_{f}\left[\sigma_{1}|\cdots| \sigma_{r-1}\right] \times \mu_{F}\left[\sigma_{1}\right]},
$$

owing to (1.6.2),

$$
=\sum\left(\int w_{1} \cdots w_{i}\right)_{\mu_{F}\left[\sigma_{1}|\cdots| \sigma_{s-1}\right]} \times\left(\int w_{i+1} \cdots w_{r} v\right)_{\mu_{F}\left[\sigma_{s}\right]} .
$$

It follows from the induction hypothesis that

$$
\left(\int w_{1} \cdots w_{r}\right)_{\mu_{F}\left[\sigma_{1}|\cdots| \sigma_{s}\right]}=\left(\int w_{1} \cdots w_{r}\right)_{\mu_{F}\left[\sigma_{1}\right] \times \cdots \times \mu_{F}\left[\sigma_{s}\right]} .
$$

Hence the lemma is proved.

COROLlary. For $s>r$,

$$
\left\langle\int w_{1} \cdots w_{r}, \mu_{F}\left[\sigma_{1}|\cdots| \sigma_{s}\right]\right\rangle=0 .
$$

Using the above corollary together with (2.5.4) and (1.6.6), we may verify the next formula

$$
\left\langle\int w_{1} \cdots w_{s}, \mu_{F}\left[\sigma_{1}|\cdots| \sigma_{s}\right]\right\rangle=\left(\int_{\sigma_{1}} w_{1}\right) \cdots\left(\int_{\sigma_{s}} w_{s}\right) .
$$


Observe that (2.5.8) implies that the map (2.5.5) preserves filtration. Moreover (2.5.9) implies that the composite map

$$
\otimes^{s}\left(A^{*-1}\right) \stackrel{(2.5 .3)}{\rightarrow} \varepsilon_{0}^{-s} \rightarrow E_{0}^{-s}
$$

sends $w_{1} \otimes \cdots \otimes w_{s}$ to the cochain given by

$$
\left[\sigma_{1}|\cdots| \sigma_{s}\right]+F_{-s-1}(C) \mapsto\left(\int_{\sigma_{1}} w_{1}\right) \cdots\left(\int_{\sigma_{1}} w_{s}\right) .
$$

LEMMA 2.5.2. Let $M$ be a differentiable space with $H_{0}(M)=Z$. If $A$ is a differential graded subalgebra of $\Lambda(M)$ with $d A^{0}=A^{1} \cap d \Lambda^{0}(M)$, then the cochain maps (2.5.3) are bijective.

For a proof of the above lemma, we simply remark that Lemma 2.2.3 makes the proof for Lemma 4.3.1 [24] also valid for the present situation.

Observe that (2.5.10) induces isomorphisms which are the composite maps

$$
\otimes^{s} H\left(A^{*-1}\right) \rightarrow E_{1}^{-s} \rightarrow E_{1}^{-s}, \quad s \geqslant 1 .
$$

The above lemma implies that the first arrow is an isomorphism. Finally (2.5.6) follows.

2.5. Proof of Theorem 2.3.1. Under the hypotheses of the theorem, we have the following additional information:

(i) Since $H^{0}\left(A^{*-1}\right)=H^{1}(A)=0$ and $\varepsilon_{1}^{-s} \approx \bigotimes^{s} H\left(A^{*-1}\right)$, the spectral sequence $\left\{\Theta_{r}, \widehat{D}_{r}\right\}$ converges to $H\left(A_{x_{0}}^{\prime}\right)$.

(ii) Similarly, $\left\{\bigotimes_{r}, \mathfrak{d}_{r}\right\}$ converges to $H^{*}(F(C) ; k)$.

(iii) Theorem A4.1 implies an isomorphism

$$
H^{*}\left(\Omega_{T} M ; k\right) \approx H^{*}(F(C) ; k) .
$$

It follows from (2.5.6) together with (a) and (b) that

$$
H\left(A_{x_{0}}^{\prime}\right) \approx H^{*}(F(C) ; k),
$$

which establishes the isomorphism (2.3.1).

If (2.3.2) holds, then the naturality of $\mu_{F}$ gives rise to a factorization of the isomorphism (2.3.1):

$$
H\left(A_{x_{0}}^{\prime}\right) \rightarrow H_{D R}(\Omega M) \rightarrow H^{*}(\Omega M ; k) \approx H^{*}\left(\Omega_{T} M ; k\right)
$$

which preserves the cup product. Hence the proof is completed.

2.6. The fundamental group. In the nonsimply connected case, the 0th cohomology group $H^{0}\left(A_{x_{0}}^{\prime}\right)$ does not, in general, determine the 0th homology group $H_{0}\left(\Omega_{T} M ; k\right)$, which can be regarded as the group algebra $k \pi_{1}\left({ }_{T} M\right)$ of the fundamental group $\pi_{1}\left({ }_{T} M\right)$ over $k$. In this section, we describe relations between $A_{x_{0}}^{\prime}$ and $\pi_{1}\left({ }_{T} M\right)$. It is necessary to explain first some group theoretical notions and facts.

Definition 2.6.1. The torsion free nilpotent residue of a group $G$ is the normal subgroup that is the intersection of the kernels of all possible epimorphisms from $G$ to a torsion free nilpotent group. A group is residually torsion free nilpotent if its torsion free nilpotent residue is trivial.

Among examples of residually torsion free nilpotent groups are free groups 
and fundamental groups of Riemann surfaces. (This follows from Baumslag's work [7].)

Let $k G$ be the group algebra of a finitely generated group $G$, and let $\widetilde{\Im}_{G}$ be the augmentation ideal of $k G$ which consists of all elements $\Sigma c_{i} g_{i}, c_{i} \in k$, $g_{i} \in G$, such that $\Sigma c_{i}=0$. It is known (Proposition 2.2.1 [30]) that the torsion free residue of $G$ consists of all $g$ such that $g-1 \in \cap_{r \geqslant 1}\left(\mathfrak{\mho}_{G}\right)^{r}$.

For simplicity, assertions in this section will be stated for a connected differentiable manifold $M$ with a finite first Betti number. See [21] and [26] for general statements or further results.

THEOREM 2.6.1. If $A$ is a differential graded subalgebra of $\Lambda(M)$ such that the inclusion $A \subset \Lambda(M)$ induces a cohomology isomorphism, then

$$
\mathfrak{E}_{r}^{-r, r} \approx \operatorname{Hom}\left(\mathfrak{\Im}^{r} / \mathfrak{\Im}^{r+1}, k\right), \quad r \geqslant 1,
$$

where $\mathfrak{\Im}=\mathfrak{\Im}_{\pi_{1}(M)}$.

Corollary 1. For $s \geqslant 0, H^{0}\left(A^{\prime}(-s)\right) \approx \operatorname{Hom}\left(k \pi_{1}(M) / \Im^{s+1}, k\right)$.

COROLlaRY 2. With respect to the pairing $H^{0}\left(A_{x_{0}}^{\prime}\right) \times k \pi_{1}(M) \rightarrow k$ via integration,

$$
H^{0}\left(A_{x_{0}}^{\prime}\right)^{\perp}=\cap_{r} \Im^{r} \text {. }
$$

Observe that $H^{0}\left(A_{x_{0}}^{\prime}\right) \subset\left(A_{x_{0}}^{\prime}\right)^{0}$. For a loop $\gamma$ at $x_{0}$, denote by $[\gamma]$ the corresponding element of $\pi_{1}(M)$.

CoROLlaRY 3. An element $[\gamma] \in \pi_{1}(M)$ belongs to the residually torsion free nilpotent residue if and only if

$$
\langle u,(\gamma)\rangle=0, \quad \forall u \in H^{0}\left(A_{x_{0}}^{\prime}\right) .
$$

The next assertion is related to Stallings' work [63]. (See also [64].)

THEOREM 2.6.2. Let $f: M \rightarrow N$ be a differentiable map with $f\left(x_{0}\right)=y_{0}$, where $N$ is also a connected differentiable manifold. If $f$ induces an isomorphism $H_{D R}^{1}(N) \approx H_{D R}^{1}(M)$ and a monomorphism $H_{D R}^{2}(N) \rightarrow H_{D R}^{2}(M)$, then $f$ induces an isomorphism $H^{0}\left(B_{y_{0}}^{\prime}\right) \approx H^{0}\left(A_{x_{0}}^{\prime}\right)$, where $A=\Lambda(M)$ and $B=\Lambda(N)$.

Finally we mention that $A_{x_{0}}^{\prime}$ and $H\left(A_{x_{0}}^{\prime}\right)$ are graded Hopf algebras. The ungraded Hopf algebra structure of $A_{x_{0}}^{\prime x_{0}}$ and that of $H\left(A_{x_{0}}^{\prime}\right)$ have been considered in [21] and [26].

2.7. Function algebra extensions by integration. Let $\pi: \tilde{M} \rightarrow M$ be the universal covering projection to a connected differentiable manifold $M$. Write $F_{0}=\pi^{*} \Lambda^{0}(M)$ and $W_{0}=\pi^{*} \Lambda^{1}(M)$. Let $F$ be an intermediate algebra between $F_{0}$ and $\Lambda^{0}(\tilde{M})$, and set $W_{F}=F W_{0}$.

We say that $F^{\prime}$ is the extension over $F$ by integration if the algebra $F^{\prime}$ is obtained from $F$ by adjoining all $f \in \Lambda^{0}(\tilde{M})$ such that $d f \in W_{F}$.

Consider the sequence $F_{0} \subset F_{1} \subset \ldots \subset F_{r_{\tilde{r}}} \subset \ldots$ such that $F_{r+1}$ is the extension over $F_{r}$ by integration, $r \geqslant 0$. Let $\tilde{F}=\cup F_{r}$. The next two theorems are stated under the assumption that $M$ is a connected differentiable manifold with $H_{*}(M)$ of finite type. 
THEOREM 2.7.1. The function algebra $\tilde{F}$ separates $\tilde{M}$ if and only if the fundamental group $\pi_{1}(M)$ is residually torsion free nilpotent.

We say that the lower central series of a group $G$ :

$$
G=G_{0} \supset G_{1} \supset \ldots \supset G_{r} \supset \ldots
$$

stablizes modulo torsion if, for $r$ sufficiently large, $G_{r} / G_{r+1}$ is finite.

THEOREM 2.7.2. The algebra $\tilde{F}$ is finitely generated over $F_{0}$ if and only if the lower central series of $\pi_{1}(M)$ stablizes modulo torsion.

The above theorems have been proved in [30]. These theorems seem to indicate the possibility of extending the classical Picard-Vessiot theory to differentiable manifolds. The usual Picard-Vessiot theory treats functions of one variable. In Kolchin's differential algebraic version [45], there are theorems relating extensions by integrals and nilpotent Galois groups.

\section{Chapter III. Loop Space Homology}

We are going to present a method for computing the loop space homology. The method provides the $E^{1}$ term of the spectral sequence of the cobar construction with a new differential, which will induce the differentials of subsequent terms $E^{r}, r>1$. A generalized notion of linear connections [44] and a differentiable version of twisting cochains [11], [39] will be used. Theorems 3.4.1 and 3.3.3 constitute the basis for this method.

Our present version of the method (of formal power series connections) was introduced in [24] and improved in [27] and [30]. It should be mentioned that our work in this respect can be traced back to [13], which is related to an early work of Magnus [49].

3.1. An introduction to the method. Consider a simply connected manifold $M$. Choose for $H_{*}(M ; k)$ a basis $1, \hat{z}_{1}, \ldots, \hat{z}_{m}$ so that $\hat{z}_{i} \in H_{p_{i}}(M ; k), p_{i}>0$. Let $k[X]=k\left[X_{1}, \ldots, X_{m}\right]$ be the graded free associative algebra generated by the indeterminates $X_{1}, \ldots, X_{m}$ with $\operatorname{deg} X_{i}=p_{i}-1$.

Write $\Lambda(M)[X]=\Lambda(M) \otimes k[X]$, which can be regarded as the free associative algebra generated by $X_{1}, \ldots, X_{m}$ over $\Lambda(M)$. The exterior differentiation $d$ and the operator $J$ extend from $\Lambda(M)$ to $\Lambda(M)[X]$ by acting on coefficients. Thus, for example, $d\left(w X_{1} X_{2}\right)=d w X_{1} X_{2} ; J\left(w X_{1} X_{2}\right)=$ $(J w) X_{1} X_{2}$.

By a derivation of $k[X]$, we mean a linear endomorphism $\partial$ of the graded vector space $k[X]$ of degree -1 such that, for homogeneous elements $u$ and $v$ of $k[X]$,

$$
\partial(u v)=(\partial u) v+(-1)^{\operatorname{deg} u} u \partial v .
$$

Thus $\partial$ is uniquely determined by $\partial X_{1}, \ldots, \partial X_{m}$. We may extend $\partial$ to a derivation of $\Lambda(M)[X]$ over $\Lambda(M)$.

The next assertion will follow as a corollary of Theorem 3.4.1.

THEOREM 3.1.1. Let $\partial$ be a derivation of $k[X]$ and let

$$
\omega=\sum w_{i} X_{i}+\sum w_{i j} X_{i} X_{j}+\cdots
$$

be an element of $\Lambda(M)[X]$ such that $w_{1}, \ldots, w_{m}$ are closed forms on $M$ (whose 
de Rham cohomology classes are) dual to the basis $\hat{z}_{1}, \ldots, \hat{z}_{m}$. If

$$
\partial \omega+d \omega-J \omega \wedge \omega=0,
$$

then $(k[X], \partial)$ is a differential graded algebra (whose differential $\partial$ is of degree $-1)$, and there is an isomorphism of graded algebras

$$
H_{*}(\Omega M ; k) \approx H(k[X], \partial),
$$

which is induced by the chain map

$$
\Theta: C_{*}(\Omega M) \rightarrow k[X]
$$

such that

$$
\begin{aligned}
c \mapsto\langle 1, c\rangle & +\sum\left\langle\int w_{i}, c\right\rangle X_{i}+\sum\left\langle\int w_{i} w_{j}+w_{i j}, c\right\rangle X_{i} X_{j} \\
& +\sum\left\langle\int w_{i} w_{j} w_{k}+w_{i} w_{i j}+w_{i j} w_{k}+w_{i j k}, c\right\rangle X_{i} X_{j} X_{k}+\cdots
\end{aligned}
$$

Remark. If $H_{*}(M)$ is free, we may choose $\hat{z}_{1}, \ldots, \hat{z}_{m}$ to represent an integral basis for $H_{*}(M)$. Suppose that

$$
\partial X_{k}=\sum c_{i j}^{k} X_{i} X_{j}, \quad c^{\prime} s \in Z, 1 \leqslant k \leqslant m .
$$

According to Theorem 3.6.1, if $H(Z[X], \partial)$ is torsion free, then there is an isomorphism of rings

$$
H_{*}(\Omega M) \approx H(Z[X], \mathrm{\partial}) .
$$

EXAMPLE 3.1.1. Let $X$ be an indeterminate corresponding to the fundamental class $\hat{z} \in H^{n}\left(S^{n}\right), n>1$. Then $k[X]$ is the usual polynomial algebra of a single generator of degree $n-1$. Let $w$ be an $n$-form on $S^{n}$ with $\int_{S^{n} w}=1$. Let $\omega=w X$ and let $\partial$ be the trivial derivation of $k[X]$ such that $\partial X=0$. Then (3.1.2) holds, and there is an isomorphism of rings $\Theta_{*}: H_{*}\left(\Omega S^{n}\right) \approx$ $Z[X]$. (This ring isomorphism was first determined by Bott and Samelson [9].)

Let $c \in C_{n-1}\left(\Omega S^{n}\right)$ be a cycle such that $\hat{c}$ is the generator of the ring $H_{*}\left(\Omega S^{n}\right)$ with $\Theta_{*}(\hat{c})=X$. We may demand that $c$ is an integral linear combination of cubes of $\Omega M \subset P(M)$, each of which starts and ends smoothly so that $c^{r}=c \times \ldots \times c(r$ times $)$ is well defined. Write

$$
u_{r}=\int w \cdots w \quad(r \text { times }) .
$$

Since $\Theta_{*}$ is a ring isomorphism, we obtain

$$
\begin{aligned}
X^{r} & =\Theta_{*}\left(\hat{c}^{r}\right)=\Theta\left(c^{r}\right) \\
& =\left\langle 1, c^{r}\right\rangle+\left\langle\int w, c^{r}\right\rangle X+\left\langle\int w w, c^{r}\right\rangle X^{2}+\ldots=\left\langle u_{r}, c^{r}\right\rangle X^{r}
\end{aligned}
$$

so that $\left\langle u_{r}, c^{r}\right\rangle=1$. Therefore $1, \int_{x_{0}}^{x_{0} w}, \int_{x_{0}}^{x_{0} w w}, \cdots$ represent integral cohomology classes dual to $1, \hat{c}, \hat{c}^{2}, \ldots$

EXAMPLE 3.1.2. The complex projective $n$-space $C P^{n}, n \geqslant 1$, has an integral homology basis $1, \hat{z}_{1}, \ldots, \hat{z}_{n}$, whose dual cohomology basis is represented by 
$1, w, \ldots, w^{n}=w \wedge \cdots \wedge w\left(n\right.$-fold), where $w$ is a closed 2-form on $C P^{n}$. Choose $\omega$ and $\partial$ such that $\omega=\Sigma_{1 \leqslant i \leqslant n} w^{i} X_{i}$ and $\partial X_{j}=\Sigma_{1 \leqslant i<j} X_{i} X_{j-i}, 1<j \leqslant$ $n$, with $\partial X_{1}=0$. Observe that (3.1.2) holds. Thus

$$
H_{*}\left(\Omega C P^{n} ; k\right) \approx H\left(k\left[X_{1}, \ldots, X_{n}\right], \partial\right) .
$$

As a matter of fact, one may verify that $\partial Z\left[X_{1}, \ldots, X_{n}\right]$ is a direct summand of $Z\left[X_{1}, \ldots, X_{n}\right]$ so that there is a ring isomorphism

$$
H_{*}\left(\Omega C P^{n}\right) \approx H\left(Z\left[X_{1}, \ldots, X_{n}\right], \partial\right) .
$$

(The homology of $\Omega C P^{n}$ is known. See Ganea [38].)

EXAMPLE 3.1.3. Let $M$ be an $(n-1)$-connected closed differentiable $2 n$ manifold, $n \geqslant 2$. Choose a basis for $H_{*}(M ; k)$, which gives rise to indeterminates $X_{1}, \ldots, X_{b}, X_{b+1}$, where $b=b_{n}$ is the middle Betti number, and $\operatorname{deg} X_{b+1}=2 n-1$. Choose closed $n$-forms $w_{1}, \ldots, w_{b}$ and a closed $2 n$-form $w_{b+1}$ so that they represent the dual cohomology basis. For $i, j=1, \ldots, b$, there exist $(2 n-1)$-forms $w_{i j}$ so that

$$
J w_{i} \wedge w_{j}=-c_{i j} w_{b+1}+d w_{i j}, \quad c_{i j} \in k .
$$

Set $\partial X_{1}=\cdots=\partial X_{b}=0, \partial X_{b+1}=\sum_{1 \leqslant i j \leqslant b} c_{i j} X_{i} X_{j}$, and $\omega=\Sigma_{1 \leqslant i \leqslant b+1} w_{i} X_{i}+$ $\Sigma_{1 \leqslant i j \leqslant b} w_{i j} X_{i} X_{j}$ and observe that (3.1.2) holds. Hence

$$
H_{*}(\Omega M ; k) \approx H\left(k\left[X_{1}, \ldots, X_{b+1}\right], \partial\right) .
$$

EXAMPLE 3.1.4. Let $M$ be a simply connected closed differentiable 5-manifold. Let $b=b_{2}=b_{3}$ be the middle Betti number. Choose a basis for $H_{*}(M ; k)$ and let $X_{1}, \ldots, X_{2 b+1}$ be corresponding indeterminates with $\operatorname{deg} X_{i}$ $=1$ for $1 \leqslant i \leqslant b,=2$ for $b<i \leqslant 2 b$, and $=3$ for $i=2 b+1$. Choose closed forms $w_{1}, \ldots, w_{2 b+1}$, which represent the dual cohomology basis. Write $p_{i}=\operatorname{deg} w_{i}$. For $1 \leqslant i, j \leqslant b$, there exist 3-forms $w_{i j}$ so that $d w_{i j}-J w_{i}$ $\wedge w_{j}=0$. When $p_{i}+p_{j}=5$, let $w_{i j}$ be a 4-form with

$$
d w_{i j}-J w_{i} \wedge w_{j}=c_{i j} w_{2 b+1}, \quad c_{i j} \in k .
$$

For $1 \leqslant i, j, k \leqslant b, J w_{i} \wedge w_{j k}+J w_{i j} \wedge w_{k}$ is a 5-form representing a Massey product so that, for some 4-form $w_{i j k}$,

$$
d w_{i j k}-J w_{i} \wedge w_{j k}-J w_{i j} \wedge w_{k}=c_{i j k} w_{2 b+1}, \quad c_{i j k} \in k .
$$

Set

$$
\omega=\sum_{1<i<2 b+1} w_{i} X_{i}+\sum_{p_{i}+p_{j} \leqslant 5} w_{i j} X_{i} X_{j}+\sum_{1 \leqslant i, j, k \leqslant b} w_{i j k} X_{i} X_{j} X_{k}
$$

and

$$
\partial X_{2 b+1}=\sum_{p_{i}+p_{j} \leqslant 5} c_{i j} X_{i} X_{j}+\sum_{1 \leqslant i, j, k \leqslant b} c_{i j k} X_{i} X_{j} X_{k}
$$

with $\partial X_{1}=\cdots=\partial X_{2 b}=0$. Then (3.1.2) holds, and

$$
H_{*}(\Omega M ; k) \approx H\left(k\left[X_{1}, \ldots, X_{2 b+1}\right], \partial\right) .
$$

3.2. Transport. In order to give a full account of Theorem 3.1.1, the free associative algebra $k[X]$ needs to be completed to a noncommutative formal 
power series algebra $k[[X]]$, whose elements are infinite formal sums of the type

$$
a_{0}+\sum a_{i} X_{i}+\cdots+\sum a_{i_{1} \cdots i_{r}} X_{i_{1}} \cdots X_{i_{r}}+\cdots
$$

with $a$ 's in $k$.

For this section, the indeterminates $X_{1}, \ldots, X_{m}$ will have no attached geometrical significance. If $M$ is a differentiable space, let $\Lambda(M)[[X]]$ be the completion of $\Lambda(M)[X]$, i.e. the noncommutative formal power series algebra of the indeterminates $X_{1}, \ldots, X_{m}$ over $\Lambda(M)$.

We consider also the algebra $\Lambda(P(M))[[X]]$. The Poincare operator $\int^{\prime}$, as given by (1.5.3), can be extended to a map

$$
\int^{\prime}: \Lambda(P(M))[[X]] \rightarrow \Lambda(P(M))[[X]]
$$

by acting on coefficients. Recall the map $p_{t}: P(M) \rightarrow M, 0 \leqslant t \leqslant 1$, given by $\gamma \mapsto \gamma(t)$. Extend also $p_{t}^{*}: \Lambda(M) \rightarrow \Lambda(P(M))$ to

$$
p_{t}^{*}: \Lambda(M)[[X]] \rightarrow \Lambda(P(M))[[X]] \text {. }
$$

If $u_{1}, \ldots, u_{r} \in \Lambda(M)[[X]]$, we likewise define

$$
\int u_{1} \cdots u_{r}=\int^{\prime}\left(J \int u_{1} \cdots u_{r-1}\right) \wedge p_{1}^{*} u_{r}
$$

The differential formula in Proposition 1.5.1 becomes also valid for $\Lambda(P(M))[[X]]$. So do the formulas (1.6.1)-(1.6.6).

DEFINITION 3.2.1. A formal (power series) connection on a differentiable space $M$ is an element of $\Lambda(M)[[X]]$ of the type

$$
\omega=\sum w_{i} X_{i}+\cdots+\sum w_{i_{1} \cdots i_{r}} X_{i_{1}} \cdots X_{i_{r}}+\cdots
$$

where the coefficients are forms of positive degree on $M$. The curvature of $\omega$ is the element $\kappa=d \omega-J \omega \wedge \omega$ of $\Lambda(M)[[X]]$.

Let the symbol $\omega^{r}$ stand for $\omega \cdots \cdots\left(r\right.$ times). Let the Poincaré operator $\int^{\prime}$ act on the coefficients of each element of $\Lambda(P(M))[X]$. Define $\int \omega=\int^{\prime} p_{1}^{*} \omega$ and, for $r>1$,

$$
\int \omega^{r}=\int^{\prime}\left(J \int \omega^{r-1}\right) \wedge p_{1}^{*} \omega .
$$

DEFINITION 3.2.2. The transport of a formal connection $\omega$ is the element

$$
T=1+\int \omega+\int \omega^{2}+\cdots+\int \omega^{r}+\cdots
$$

of $\Lambda(P(M))[[X]]$.

Write the transport $T$ in the form of

$$
T=1+\sum T_{i} X_{i}+\sum T_{i j} X_{i} X_{j}+\cdots .
$$

Verify that 


$$
\begin{aligned}
T_{i} & =\int w_{i}, \quad T_{i j}=\int\left(w_{i} w_{j}+w_{i j}\right), \\
T_{i j k} & =\int\left(w_{i} w_{j} w_{k}+w_{i} w_{j k}+w_{i j} w_{k}+w_{i j k}\right), \ldots \text { etc. }
\end{aligned}
$$

Thus our present definition of the transport coincides with that given in $\$ 2.1$ [27]. Observe also that $T$ is the unique solution of the equation

$$
T=1+\int^{\prime}\left(J T \wedge p_{1}^{*} \omega\right) \text {. }
$$

REMARK. This equation can be used to define more general connections in the sense of [27].

By making use of Proposition 1.5.2 and its extension to $\Lambda(P(M))[[X]]$, one may verify that

$$
\begin{aligned}
d T= & -\int \kappa+\left(-\int \kappa \omega+\int J \omega \kappa\right)+\cdots \\
& +\sum_{i+j=r-1} \int(J \omega)^{i} \kappa \omega^{j}+\cdots-p_{0}^{*} \omega \wedge T+J T \wedge p_{1}^{*} \omega .
\end{aligned}
$$

Let $\alpha: U \rightarrow P(M)$ be a compact plot. Define

$$
\langle T, \alpha\rangle=\langle 1, \alpha\rangle+\sum\left\langle T_{i}, \alpha\right\rangle X_{i}+\sum\left\langle T_{i j}, \alpha\right\rangle X_{i} X_{j}+\cdots .
$$

It follows from (1.6.6) and its extension to $\Lambda(P(M))[[X]]$ that, if $\alpha^{\prime}: U^{\prime} \rightarrow$ $P(M)$ is another compact plot such that the product plot $\alpha \times \alpha^{\prime}$ is well defined, then

$$
\left\langle T, \alpha \times \alpha^{\prime}\right\rangle=\langle T, \alpha\rangle\left\langle T, \alpha^{\prime}\right\rangle .
$$

The above formula gives rise to a multiplication preserving "generalized holonomy map"

$$
\Theta: C_{*}(\Omega M) \rightarrow k[[X]]
$$

given by $c \mapsto\langle T, c\rangle$, where $C_{*}(\Omega M)$ is the normalized cubical chain complex of $\Omega M$.

Material in this section can be found in [24] with a slightly different presentation.

3.3. Twisting cochains. Our problem in this section is to equip $k[[X]]$ with a suitable differential $\partial$ so that the map $\Theta$ becomes a chain map.

Recall that every element of $k[[X]]$ can be written as

$$
a=a_{0}+\sum a_{i} X_{i}+\cdots+\sum a_{i_{1} \cdots i_{r}} X_{i_{1}} \cdots X_{i_{r}}+\cdots .
$$

Let $\Im$ be the augmentation ideal of $k[[X]]$, which consists of all elements $a$ such that $a_{0}=0$. The sth power $\Im^{s}$ of $\lesssim$ consists of all $a$ such that $a_{i_{1}} \ldots i_{r}=0$ for $r<s$.

A derivation $\partial$ of $k[[X]]$ is a linear endomorphism of $k[[X]]$ satisfying the following conditions:

(a) If $u$ and $v$ are homogeneous elements of $k[[X]]$, then

$$
\partial(u v)=(\partial u) v+(-1)^{\operatorname{deg} u} u \partial v .
$$


(b) For $1 \leqslant i \leqslant m, \partial X_{i} \in \mathfrak{\Im}^{2}$.

(c) For any $a \in k[[X]]$ as given in (3.3.1),

$$
\partial a=\sum a_{i} \partial X_{i}+\cdots+\sum a_{i_{1} \cdots i_{r}} \partial\left(X_{i_{1}} \cdots X_{i_{r}}\right)+\cdots
$$

Every derivation of $k[[X]]$ can be extended to a derivation of $\Lambda(M)[[X]]$ in the obvious sense.

THEOREM 3.3.1. Let $\omega$ be a formal connection on a differentiable space $M$, and $\kappa$, its curvature. If $\partial$ is a derivation of $k[[X]]$ such that

$$
\partial \omega+\kappa=0
$$

holds, then

$$
d T=\partial T-p_{0}^{*} \omega \wedge T+J T \wedge p_{1}^{*} \omega .
$$

Proof. Substitute $\kappa$ by $-\partial \omega$. Then (3.2.4) becomes (3.3.3).

Corollary. Under the same hypothesis as the theorem, if $\left.T\right|_{\Omega M}$ denotes the restriction of $T$ on $\Omega M$, then

$$
\left.d T\right|_{\Omega M}=\left.\partial T\right|_{\Omega M}
$$

REMARK. The condition (3.3.2) can be written in the form of (3.1.2) which is a differentiable version of the condition for a twisting cochain [11], [39]. Strictly speaking, $\omega$ may not be called a twisting cochain at this moment, because $\partial \partial$ is not necessarily trivial.

From now on, we assume that $M$ is a differentiable space with $H_{0}(M)=Z$ and $H_{*}(M)$ of finite type. Let $1, \hat{z}_{1}, \ldots, \hat{z}_{m}$ be a basis of $H_{*}(M ; k)$ with $\hat{z}_{\mathrm{i}} \in H_{p_{i}}(M ; k)$. For simplicity, assume that $m$ is finite. The corresponding indeterminates $X_{1}, \ldots, X_{m}$ are such that $\operatorname{deg} X_{i}=p_{i}-1$.

DEFINITION 3.3.1. A formal homology connection on $M$ is a pair $(\omega, \partial)$ consisting of a formal connection $\omega$ as given by (3.2.1) and a derivation $\partial$ of $k[[X]]$ satisfying the twisting cochain condition (3.3.2) such that

(a) $w_{1}, \ldots, w_{m}$ are closed forms dual to $\hat{z}_{1}, \ldots, \hat{z}_{m}$;

(b) $\operatorname{deg} w_{i_{1} \cdots i_{r}}=p_{1}+\cdots+p_{r}-r+1, r \geqslant 1$.

Proposition 3.3.2. If $(\omega, \partial)$ is a formal homology connection on $M$, then $\partial \partial=0$.

The above assertion follows from the fact that the associated "generalized holonomy map" $\Theta$ has a dense image in $k[[X]]$ and the fact that $\partial \partial\langle T, c\rangle=$ $\langle T, \partial \partial c\rangle=0, c \in C_{*}(\Omega M)$.

Let $A$ be a differential graded subalgebra of $\Lambda(M)$. We shall consider a direct sum decomposition of $A$ of the type

$$
A=\mathfrak{g} \oplus d \mathfrak{B} \oplus \mathfrak{B}
$$

where (a) $\mathfrak{S}$ is a graded subspace of closed form of $A$; (b) $\mathfrak{B}$ is a graded subspace of $A$ containing no closed forms other than 0 . Then $\mathfrak{S} \approx H(A)$.

THeorem 3.3.3. Let $M$ be a differentiable space as described above. Let $A$ be a differential graded subalgebra of $\Lambda(M)$ such that $H(A) \approx H^{*}(M ; k)$ via 
integration. Then, for every direct sum decomposition of $A$ of the type (3.3.5), there exists a unique formal homology connection $(\omega, \partial)$ with $\omega$ being given as in (3.2.1) such that $w_{i} \in \mathfrak{S}$ and $w_{i j}, w_{i j k}, \ldots \in \mathfrak{W}, i, j, k, \ldots=1, \ldots, m$.

The proof for Theorem 1.3.1 [30] is also valid for the above theorem, which is in a more general setting.

See also the remarks to Theorem 1.3.1 and Proposition 1.3.3 [30] regarding consequences of the uniqueness property of $(\omega, \partial)$. In particular, $\omega$ and $\kappa$ are both "Lie elements".

Let $\left[w_{i}\right]$ denote the cohomology class of the closed form $w_{i}$. Verify that the twisting cochain condition (3.3.2) makes

$$
\partial X_{i}=\sum c_{j k}^{i} X_{j} X_{k}+\cdots,
$$

where the coefficients $c$ 's are determined by cup products $\left[J w_{j} \wedge w_{k}\right]=$ $\sum c_{j k}^{i}\left[w_{i}\right]$.

3.4. A theorem on loop space homology. Let $(\omega, \partial)$ be a given formal homology connection on $M$. Make $k[[X]]$ graded by assigning $\operatorname{deg} X_{i}=p_{i}-$ $1,1 \leqslant i \leqslant m$. In (3.2.1), we have consequently

$$
\operatorname{deg} w_{i_{1} \cdots i_{r}}=1+\operatorname{deg} X_{i_{1}} \cdots X_{i_{r}} \text {. }
$$

The twisting cochain condition (3.3.2) forces the derivation $\partial$ to be a graded map of degree -1 .

Equip $k[[X]]$ with an ascending filtration by powers of the augmentation ideal $\mathfrak{J}$. Then $(k[[X]], \partial)$ is a filtered chain complex, whose spectral sequence $\left\{\mathfrak{E}^{r}, \mathfrak{D}^{r}\right\}_{r \geqslant 0}$ is such that $\mathfrak{F}_{-s}^{0}=\mathfrak{J}^{s} / \mathfrak{\Im}^{s+1}, s \geqslant 1, \mathfrak{F}_{0}^{0}=k[[X]] / \mathfrak{I} \approx k$, and $\mathfrak{G}_{s}^{0}=0$ for $s>0$. Since $\partial X_{i} \in \mathfrak{J}^{2}$, we have

$$
\mathbb{G}_{-s}^{1}=\mathfrak{\Im}^{s} / \mathfrak{J}^{s+1} \approx \otimes^{s} \mathfrak{\Im} / \mathfrak{\mho}^{2} \approx \otimes^{s} H_{*-1}(M ; k), \quad s \geqslant 1 .
$$

If each $X_{i}$ is of degree $>0$, then the free algebra $k[X]$, as a subalgebra of $k[[X]]$, is stable under $\partial$ and is therefore also a filtered chain complex. The associated spectral sequence, being isomorphic to $\left\{\ll r, b^{r}\right\}_{r \geqslant 0}$, will be also denoted by $\left\{\Theta^{r}, \mathbb{D}^{r}\right\}_{r} \geqslant 0$. In this case, the spectral sequence converges to $\boldsymbol{H}(k[X], \partial)$.

THEOREM 3.4.1. Let $M$ be a topological differentiable space with a base point $x_{0}$ and having the following properties:

(a) The inclusions $\Delta(M)_{x_{0}} \subset \Delta(M) \subset \Delta\left({ }_{T} M\right)$ induce isomorphisms

$$
H\left(\Delta(M)_{x_{0}}\right) \approx H_{*}(M) \approx H_{*}\left(T^{M}\right) .
$$

(b) $H_{*}(M)$ is of finite type.

If $(\omega, \partial)$ is a formal homology connection on $M$ and if $\left\{\ll r, \mathbb{b}^{r}\right\}$ is the spectral sequence of $F\left(\Delta(M)_{x_{0}}\right)$ as defined in $\$ 2.5$, then the chain map

$$
F\left(\Delta(M)_{x_{0}}\right) \stackrel{\mu_{r}}{\rightarrow} C_{*}(\Omega M) \stackrel{\Theta}{\rightarrow} k[[X]]
$$

induces isomorphisms

$$
E^{r} \otimes_{z} k \approx \Subset r, \quad r \geqslant 1 .
$$


Proof. Use an argument similar to that in the proof of Lemma 2.5.1 and show that the chain map (3.4.1) preserves multiplication. Since $\mu_{F}[\sigma] \in \mathfrak{J}$, the chain map (3.4.1) preserves filtration and gives rise to spectral sequence homomorphisms $E^{r} \otimes_{z} k \rightarrow \mathbb{E}^{r}, r \geqslant 1$.

When $r=1$, we have

$$
E_{-s}^{1} \otimes_{Z} k \approx \otimes^{s} H_{*-1}(M ; k) \approx \Im^{s} / \Im^{s+1}=\Im_{-s}^{1}, \quad s \geqslant 1 .
$$

The theorem then follows.

COROLlary. Under the same hypotheses as the theorem, if ${ }_{T} M$ is simply connected, then there is an isomorphism of algebras

$$
H_{*}\left(\Omega_{T} M ; k\right) \approx H_{*}(k[X], \partial) .
$$

DEFINITION 3.4.1. A formal homology connection $(\omega, \partial)$ is quadratic if $\partial X_{i}=\sum c_{j k}^{i} X_{i} X_{j}, 1 \leqslant i \leqslant m$.

If $(\omega, \partial)$ is quadratic, then the differential $\partial$ is determined by the cohomology cup product according to (3.3.6), and the chain complex $(k[[X]], \partial)$ is therefore intrinsically defined for the differentiable space $M$.

Lemma 3.4.2. Under the same hypotheses as Theorem 3.3.3, if a direct sum decomposition $A=\mathfrak{S} \oplus d \mathfrak{B} \oplus \mathfrak{B}$ of the type (3.3.5) is such that $d \mathfrak{B} \oplus \mathfrak{B}$ is an ideal of the differential graded algebra $A$, then the uniquely associated formal homology connection $(\omega, \partial)$ is quadratic.

Proof. For $w \in A$, let $\bar{w}$ denote the coset $w+(d \mathfrak{B} \oplus \mathfrak{B})$ and write

$$
\bar{\omega}=\sum \bar{w}_{i} X_{i}+\sum \bar{w}_{i j} X_{i} X_{j}+\cdots=\sum \bar{w}_{i} X_{i} .
$$

Since $\partial \omega+\kappa=0$, we have

$$
\sum \bar{w}_{i} \partial X_{i}=\partial \bar{\omega}=J \bar{\omega} \wedge \bar{\omega}=\sum c_{j k}^{i} \bar{w}_{i} X_{j} X_{k} .
$$

Hence $\partial X_{i}=\sum c_{j k}^{i} X_{j} X_{k}$.

The simplest case for which the lemma applies is that of $A=\mathfrak{E}$. This case includes compact symmetric spaces. All but one of the examples in $\$ 3.1$ exhibit manifolds having a quadratic formal homology connection. The above lemma applies to those examples. In the next example, $m$ is no longer finite.

EXAMPLE 3.4.1. Consider $\Omega S^{n}, n>2$, as a topological differentiable space. Recall the isomorphisms (A2.6). Use Lemma 1.3.1 to establish the canonical chain equivalence

$$
\Delta\left(\Omega S^{n}\right)_{\eta_{x_{0}}} \rightarrow \Delta\left(r^{\Omega} S^{n}\right)_{\eta_{i_{0}}}
$$

where $\eta_{x_{0}}$ is the constant loop at the base point $x_{0} \in S^{n}$.

According to Example 2.3.1, $\Lambda\left(\Omega S^{n}\right)$ has a differential graded subalgebra $A_{x_{0}}^{\prime}$ with a basis consisting of closed forms $1, u_{1}, \ldots, u_{r}, \ldots$, where

$$
u_{r}=\int_{x_{0}}^{x_{0}} w \cdots w \quad(r \text { times }) \text { and } J u_{r} \wedge u_{s}=n_{r s} u_{r+s} .
$$

There is an isomorphism $A_{x_{0}}^{\prime} \approx H^{*}\left(\Omega S^{n} ; k\right)$.

Let $X_{1}, X_{2}, \ldots$ be indeterminates corresponding to the dual basis of $H_{*}\left(\Omega S^{n} ; k\right)$ so that $\operatorname{deg} X_{i}=(n-1) i-1$. Let $(\omega, \partial)$ be the quadratic formal 
homology connection such that $\omega=\sum_{i \geqslant 1} u_{i} X_{i}$ and $\partial X_{i}=\sum_{r+s=i} n_{r s} X_{r} X_{s}, i \geqslant$ 1. Then the Corollary to Theorem 3.4.1 applies, and there is an isomorphism of algebras

$$
H_{*}\left({ }_{T} \Omega \Omega S^{n} ; k\right) \approx H\left(k\left[X_{1}, X_{2}, \ldots\right], \partial\right) .
$$

Take note that ${ }_{T} \Omega \Omega S^{n}$ is of the same homotopy type as the twice iterated continuous loop space $\Omega \Omega_{T} S^{n}$.

For compact Kähler manifolds [69], there is a remarkable result in [31], which can be translated into our setting.

Theorem 3.4.3 (Deligne-Griffiths-Morgan-Sullivan). Every compact Kähler manifold $M$ has a quadratic formal connection.

Proof. Let $\Lambda(M)$ be the de Rham complex of complex valued forms on $M$. The exterior differential can be written as a sum $d=d^{\prime}+d^{\prime \prime}$ so that, for $f \in \Lambda^{0}(M), d^{\prime} f$ is a $(1,0)$-form. Recall the $d^{\prime} d^{\prime \prime}$-lemma:

$$
\operatorname{Ker} d^{\prime} \cap \operatorname{Im} d^{\prime \prime}=\operatorname{Ker} d^{\prime \prime} \cap \operatorname{Im} d^{\prime}=\operatorname{Im} d^{\prime} d^{\prime \prime} .
$$

Set $A=\operatorname{Ker} d^{\prime}$, which is a differential graded subalgebra of $\Lambda(M)$. Using the $d^{\prime} d^{\prime \prime}$-lemma, verify that the inclusion induces an isomorphism $H(A) \approx$ $H_{D R}^{*}(M)$.

On the other hand, the $d^{\prime} d^{\prime \prime}$-lemma also implies that $d A=d^{\prime \prime} A \subset d^{\prime} \Lambda(M)$ so that $d^{\prime} \Lambda(M)$ is a differential graded ideal of $A$. The induced differential of the quotient $A / d^{\prime} \Lambda(M)=H\left(\Lambda(M), d^{\prime}\right)$ is trivial. The $d^{\prime} d^{\prime \prime}$-lemma is again used to verify that the quotient map induces an isomorphism $H(A) \approx$ $A / d^{\prime} \Lambda(M)$.

Let $\mathfrak{B}$ be a direct summand of $d^{\prime} \Lambda(M)$ complementary to $d A$ so that there is a direct sum decomposition $A=\mathfrak{S} \oplus d \mathfrak{B} \oplus \mathfrak{B}$ which meets the requirement of Lemma 3.4.2. Hence the theorem follows.

3.5. On the nonsimply connected case. In this section, we discuss the role of the degree 0 component of a formal homology connection in relation to the fundamental group. Since our aim is to convey a general idea, we shall assume, for the sake of simplicity, that $M$ is a connected differentiable manifold with a finitely generated fundamental group.

Let $(\omega, \partial)$ be a formal homology connection on $M$ with $\omega$ given as in (3.2.1) such that $\operatorname{deg} X_{i}=0$ for $1 \leqslant i \leqslant m_{0},=1$ for $m_{0}<i \leqslant m_{0}+m_{1}$, and $>1$ for $m_{0}+m_{1}<i \leqslant m$. Write $Y_{i}=X_{m_{0}+i}$ for $1 \leqslant i \leqslant m_{1}$. Denote by $k[[X]]_{q}$ the degree $q$ component of the graded algebra $k[[X]]$. Then $k[[X]]_{0}=$ $k\left[\left[X_{1}, \ldots, X_{m_{0}}\right]\right.$.

Write $\mathfrak{R}=\partial k[[X]]_{1}$. Since $\partial X_{i}=0,1 \leqslant i \leqslant m_{0}$, each element of $\mathfrak{R}$ is a (finite or infinite) sum of elements of the type $a \partial Y_{i} b$ with $a, b \in k[[X]]_{0}$ and $1 \leqslant i \leqslant m_{1}$.

Denote by $\omega_{0}$ the degree 0 component of the formal connection $\omega$. Then all coefficients of $\omega_{0}$ are 1-forms so that $\omega_{0}$ can be taken as a $k[[X]]_{0}$-valued 1 -form on $M$. Its curvature $\kappa_{0}=d \omega_{0}+\omega_{0} \wedge \omega_{0}$ is a $k[[X]]_{0}$-valued 2-form on $M$. The transport $T_{0}$ of the formal connection $\omega_{0}$ is precisely the degree 0 component of the transport $T$ of $\omega$. For any path $\gamma$ in $M$ write $T_{0}(\gamma)=$ $\left\langle T_{0},(\gamma)\right\rangle=\langle T,(\gamma)\rangle$.

The degree 0 component of the twisting cochain condition (3.3.2) is 


$$
\partial \omega_{1}+\kappa_{0}=0
$$

where $\omega_{1}=\Sigma_{1 \leqslant i \leqslant m_{1}} w_{m_{0}+i} Y_{i}$. Let $\bar{\omega}_{0}$ be the $k[[X]]_{0} / \mathfrak{R}$-valued 1-form obtained from the $k[[X]]_{0}$-valued 1 -form $\omega_{0}$. Then the curvature of $\bar{\omega}_{0}$ is $\bar{\kappa}_{0}=-\partial \bar{\omega}_{0}=$ 0 . This means that $\bar{\omega}_{0}$ is locally flat, and the associated holonomy map reduces to a multiplicative homomorphism

$$
\Theta_{0}: \pi_{1}(M) \rightarrow k[[X]]_{0} / \mathfrak{R}=H_{0}(k[[X]], \partial) .
$$

given by $[\gamma] \mapsto T_{0}(\gamma)+\mathfrak{R}$.

The next theorem is a corollary of Theorem 2.1.1 [30].

THEOREM 3.5.1. If $(\omega, \partial)$ is a formal homology connection on $M$, then the kernel of the above homomorphism $\Theta_{0}$ is the torsion free nilpotent residue (Definition 2.6.1) of $\pi_{1}(M)$.

Corollary 1 (Stallings). If $H_{2}(M)=0$, then $\pi_{1}(M)$ contains a free subgroup of rank $m_{0}$.

COROLlaRY 2. If $M$ has a quadratic formal homology connection and if the cup product $H^{1}(M ; k) \otimes H^{1}(M ; k) \rightarrow H^{2}(M ; k)$ is trivial, then $\pi_{1}(M)$ contains a free subgroup of rank $m_{0}$.

Under the hypotheses of both corollaries, we have $N=0$ and $\Theta_{0}: \pi_{1}(M) \rightarrow$ $k[[X]]_{0}$. The argument for the proof of Theorem 4.1 [22] will lead to the above corollaries.

COROLlary 3. If $M$ is a Riemann surface, then $\Theta_{0}$ is injective.

This corollary follows from the fact that $\pi_{1}(M)$ is residually torsion free nilpotent.

3.6. Integral homological applications. The spectral sequence of the cobar construction $F\left(\Delta(M)_{x_{0}}\right) \otimes_{Z} k$ is $\left\{E^{r} \otimes_{Z} k, D^{r} \otimes_{Z} 1\right\}$. There is a canonical $\operatorname{map} E^{r} \rightarrow E^{r} \otimes_{Z} k$.

If $E^{r}$ is $Z$-free and if $E^{r} \otimes_{Z} k=E^{r+1} \otimes_{Z} k$, then the above map is injective, and $d^{r}=0$ so that $E^{r}=E^{r+1}$. Thus, if $E^{r}$ is $Z$-free, then a collapsing $E^{r} \otimes_{z} k=E^{\infty} \otimes_{z} k$ implies a collapsing $E^{r}=E^{\infty}$.

In $\$ 3.3$, we may demand that the basis $\left\{1, \hat{z}_{1}, \ldots, \hat{z}_{m}\right\}$ of $H_{*}(M ; k)$ be integral; i.e. it is also a $Z$-module basis for the image of the canonical map $H_{*}(M) \rightarrow H_{*}(M ; k)$. Then $w_{1}, \ldots, w_{m}$ represent integral cohomology classes. In this case, the associated indeterminates $X_{1}, \ldots, X_{m}$ are said to be integral.

If $(\omega, \partial)$ is quadratic, then $Z[X]$, as a subring of $k[X]$, is stable under $\partial$, and gives rise to an integral chain complex. If $H_{*}(M)$ is free, then

$$
E^{1} \approx Z[X], \quad E^{2} \approx H(Z[X], \partial) .
$$

THEOREM 3.6.1. Under the same hypotheses as the Corollary to Theorem 3.4.1, if $(\omega, \partial)$ is a quadratic formal homology connection as described above and if both $H_{*}(M)$ and $H\left(Z\left[X_{1}, \ldots, X_{m}\right], \partial\right)$ are $Z$-free, then

$$
H_{*}\left(\Omega_{T} M\right) \approx H\left(Z\left[X_{1}, \ldots, X_{m}\right], \partial\right) .
$$

Proof. Since $(\omega, \partial)$ is quadratic, the spectral sequence of $k[X]$ has a 
collapsing $\mathbb{E}^{2}=H(k[X], \partial)=\uplus^{\infty}$. Therefore, according to Theorem 3.4.1, $E^{2} \otimes_{z} k=E^{\infty} \otimes_{z} k$ which implies $E^{2}=E^{\infty}$, and the theorem is proved.

This theorem has been applied in Examples 3.1.1 and 3.1.2. The next theorem illustrates how a formal homology connection determines certain "leading" terms of the spectral sequence $\left\{E^{r}, d^{r}\right\}$, which are sometimes geometrically meaningful.

THEOREM 3.6.2. Under the hypothesis of Theorem 3.6.1, let $(\omega, \partial)$ be a formal homology connection, whose indeterminates are chosen to be integral. Let $r \geqslant 2$ and $q \geqslant 0$. If $H_{q^{\prime}}(M)$ is $Z$-free for $q^{\prime} \leqslant q$, and if $\operatorname{deg} X_{i} \leqslant q$ implies

$$
\partial X_{i} \in \mathfrak{s}^{r} \text {, }
$$

then, for each indeterminate $X_{k}$ of degree $q$,

$$
\partial X_{k}=\sum c_{i_{1} \cdots i_{r}}^{k} X_{i_{1}} \cdots X_{i_{r}} \bmod \mathfrak{\Im}^{r+1}
$$

such that each coefficient $c_{i_{1}}^{k} \ldots i_{r}$ is an integer. Moreover $E_{-r, r+q-1}^{r}$ is isomorphic with the quotient of the free $Z$-module on the set of monomials $X_{i_{1}} \cdots X_{i_{r}}$ with $\operatorname{deg} X_{i_{1}} \cdots X_{i_{r}}=q-1$ over the submodule spanned by all $\sum c_{i_{1}}^{k} \cdots i_{r} X_{i_{1}} \cdots X_{i_{r}}$ with $\operatorname{deg} X_{k}=q$.

Proof. The condition (3.6.1) implies

$$
\left(\partial \Im^{s}\right)_{q^{\prime}} \subset\left(\Im^{s+r-1}\right)_{q^{\prime}-1}, \quad q^{\prime}<q,
$$

so that

$$
\mathfrak{\mho}_{-s, s+q^{\prime}}^{1}=\cdots=\mathfrak{\mho}_{-s, s+q^{\prime}}^{r-1}=\left(\Im^{s} / \Im^{s+1}\right)_{q^{\prime}}, \quad q^{\prime}<q
$$

and

$$
\mathbb{G}_{-1,1+q}^{1}=\cdots=\mathbb{\mho}_{-1,1+q}^{r-1}=\left(\mathfrak{\Im} / \mathfrak{\Im}^{2}\right)_{q} \text {. }
$$

Since $H_{q^{\prime}}(M)$ is $Z$-free for $q^{\prime} \leqslant q$, so is $E_{-s, s+q^{\prime}}^{1}$. A refinement of the argument at the beginning of this section leads to

$$
E_{-s, s+q^{\prime}}^{1}=\cdots=E_{-s, s+q^{\prime}}^{r-1}, \quad q^{\prime}<q,
$$

and

$$
E_{-1,1+q}^{1}=\cdots=E_{-1,1+q}^{r-1} .
$$

In the commutative diagram

$$
\begin{aligned}
& E_{-1,1+q}^{r-1} \longrightarrow \mathcal{E}_{-1,1+q}^{r-1} \approx\left(\Im / \Im^{2}\right)_{q} \\
& \downarrow^{r-1} \quad \downarrow^{r-1} \\
& E_{-r, r+q-1}^{r-1} \longrightarrow\left(\mathcal{F}_{-r, r+q-1}^{r-1} \approx\left(\mathcal{\Im}^{r} / \mathcal{G}^{r+1}\right)_{q-1},\right.
\end{aligned}
$$

the image of the upper horizontal arrow is spanned by all $X_{k}+\mathfrak{\mho}^{2}$ with $\operatorname{deg} X_{k}=q$, and the image of the lower horizontal arrow is spanned by all $X_{i_{1}} \cdots X_{i_{r}}+\Im^{r+1}$ with $\operatorname{deg} X_{i_{1}} \cdots X_{i_{r}}=q-1$. The theorem then follows.

ExAMPLE 3.6.1. Let $L$ be a link having two components $L_{1}$ and $L_{2}$, which are smoothly embedded circles in $S^{3}$. Let $M=S^{3}-L$. Then $H_{1}(M)$ has a 
basis $\left\{\hat{z}_{1}, \hat{z}_{2}\right\}$ where $z_{i}$ is represented by a circle around $L_{i}, i=1,2$, and $H_{2}(M)$ is free cyclic with a generator $\hat{z}_{3}$. Let $X_{1}, X_{2}, X_{3}$ be the corresponding indeterminates so that $\operatorname{deg} X_{1}=\operatorname{deg} X_{2}=0$ and $\operatorname{deg} X_{3}=1$.

Choose a direct sum decomposition of $A=\Lambda(M)$ of the type (3.3.5), which gives rise to a formal homology connection $(\omega, \partial)$ with

$$
\omega=w_{1} X_{1}+w_{2} X_{2}+w_{3} X_{3}+\cdots \text {. }
$$

According to Proposition 1.3.3 [30], $\omega$ and each $\partial X_{i}$ must be Lie elements. Let $[$,$] denote the Lie bracket. Then$

$$
\begin{aligned}
& \omega_{0}=w_{1} X_{1}+w_{2} X_{2}+w_{12}\left[X_{1}, X_{2}\right]+\cdots, \\
& \kappa_{0}=d \omega_{0}+\omega_{0} \wedge \omega_{0}=\left(d w_{12}+w_{1} \wedge w_{2}\right)\left[X_{1}, X_{2}\right]+\cdots
\end{aligned}
$$

and

$$
\partial X_{3}=c_{12}\left[X_{1}, X_{2}\right]+\cdots,
$$

where $c_{12}$ must be an integer according to Theorem 3.6.2. The condition (3.5.1) yields $\left[w_{1} \wedge w_{2}\right]=-c_{12}\left[w_{3}\right]$, which implies that the integer $c_{12}$ is, up to a difference in sign, the linking number of $L_{1}$ and $L_{2}$.

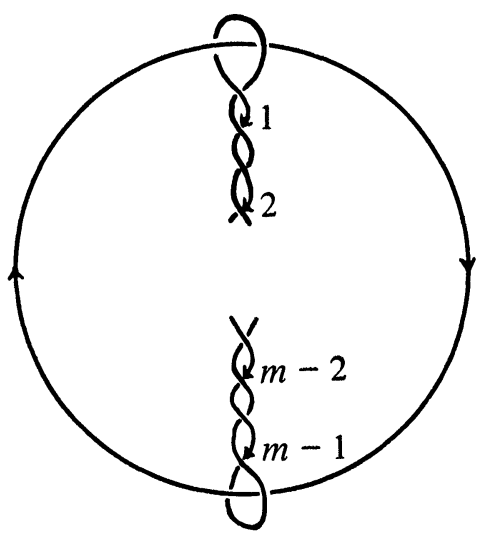

FIGURE 1

Figure 1 shows the projection of a link $L$ with $c_{12}=0$. Without giving a detailed computation, we just mention that, in this case,

$$
\partial X_{3}=(m-1)\left[\left[\left[X_{1}, X_{2}\right], X_{1}\right], X_{2}\right]+\cdots .
$$

This example indicates a need to relate Theorem 3.6.2 to Milnor's invariants [52] and Massey products [50].

\section{ChAPTER IV. BAR CONSTRUCTION AND FREE PATH FIBRATION}

Let $M$ be a differentiable space with $x_{0} \in M$. Let $A$ be a differential graded subalgebra of $\Lambda(M)$. We first show that, under mild conditions, the reduced bar construction $B(A)$ is isomorphic to $A_{x_{0}}^{\prime}$. We then consider the free path fibration $P(M) \rightarrow M \times M$ given by $\gamma \mapsto(\gamma(0), \gamma(1))$. Denote by $E_{f}$ (the total space of) the pullback via a differentiable map $f: N \rightarrow M \times M$. A subcomplex $A_{f}^{\prime}$ of $\Lambda\left(E_{f}\right)$ is constructed from $A^{\prime}$ and $\Lambda(N)$. The algebraic 
structure of $A_{f}^{\prime}$ is determined and is closely related to the two-sided bar construction [12], [60]. We state Theorem 4.3.1 which gives an isomorphism $H\left(A_{f}^{\prime}\right) \approx H^{*}\left(E_{f} ; k\right)$ when $H(A) \approx H_{D R}(M)$ and $M$ and $N$ are manifolds with $M$ simply connected and having homology of finite type.

In order to illustrate the significance of this theorem, we mention the following examples:

(a) If $f: N_{0} \times N_{1} \stackrel{\supset}{\rightarrow} M \times M$ is an inclusion, then $E_{f}$ is the space of paths from $N_{0}$ to $N_{1}$ in $M$.

(b) If $f: M \rightarrow M \times M$ is the diagonal map, then $E_{f}$ is the free loop space of M.

(c) If $f=p \times f^{\prime}: E^{\prime} \times M^{\prime} \rightarrow M \times M, p$ being a fibration, and if $E_{f^{\prime}}^{\prime}$ is the pullback of the fibration $p: E^{\prime} \rightarrow M$ via $f^{\prime}: M^{\prime} \rightarrow M$, then $E_{f^{\prime}}^{\prime}$ and $E_{f}$ are of the same homotopy type.

In particular, the last example points out that Theorem 4.3.1 provides an analytic realization of a theorem of Eilenberg and Moore [35]. (See [65] for a general discussion on fibrations and path spaces.)

4.1. The bar construction. Let $A$ be an arbitrary differential graded algebra with a differential $d$ of degree 1 such that $A^{p}=0$ for $p<0$ and $A^{0}=k$. The multiplication will be denoted by $\wedge$, and our primary example is the case of $A$ being a differential graded subalgebra of the de Rham complex of a differentiable space.

Let $W^{\prime}$ and $W^{\prime \prime}$ be respectively differential graded right and left $A$-modules. Let $w, w_{1}, w_{2}, \ldots$ denote (homogeneous) elements of $A^{+}$, and let $x$ and $y$ denote respectively elements of $W^{\prime}$ and $W^{\prime \prime}$. We use $\Lambda$ also to denote the action by $A$.

Let $B\left(W^{\prime}, A, W^{\prime \prime}\right)=\Sigma \oplus B^{r}\left(W^{\prime}, A, W^{\prime \prime}\right)$ be such that

$$
B^{r}\left(W^{\prime}, A, W^{\prime \prime}\right)=W^{\prime} \otimes\left(\otimes^{r} A^{+}\right) \otimes W^{\prime \prime}
$$

for $r>0,=W^{\prime} \otimes W^{\prime \prime}$ for $r=0$ and $=0$ for $r<0$. Thus $B^{r}\left(W^{\prime}, A, W^{\prime \prime}\right)$ is spanned by elements of the type $x\left[w_{1}|\cdots| w_{r}\right] y$, whose degree is defined to be

$$
\operatorname{deg} x+\left(-1+\operatorname{deg} w_{1}\right)+\cdots+\left(-1+\operatorname{deg} w_{r}\right)+\operatorname{deg} y .
$$

The differential $d_{B}$ is given by

$$
\begin{aligned}
d_{B}\left(x\left[w_{1}|\cdot \cdots| w_{r}\right] y\right) & =x\left[w_{1}|\cdot \cdots| w_{r}\right] y-J x \wedge w_{1}\left[w_{2}|\cdot \cdot| w_{r}\right] y \\
& +\sum_{1<i<r}(-1)^{i} J x\left[J w_{1}|\cdots| J w_{i-1}\left|d w_{i}\right| w_{i+1}|\cdots| w_{r}\right] y \\
& -\sum_{1<i<r}(-1)^{i} J x\left[J w_{1}|\cdots| J w_{i-1}^{\prime}\left|J w_{i} \wedge w_{i+1}\right| w_{i+2}|\cdots| w_{r}\right] y \\
+ & J\left(x\left[w_{1}|\cdots| w_{r-1}\right]\right) w_{r} \wedge y+J\left(x\left[w_{1}|\cdots| w_{r}\right]\right) d x .
\end{aligned}
$$

There is a filtration $F$ of $B\left(W^{\prime}, A, W^{\prime \prime}\right)$ with

$$
F^{-s} B\left(W^{\prime}, A, W^{\prime \prime}\right)=\sum_{r<s} B^{r}\left(W^{\prime}, A, W^{\prime \prime}\right) .
$$

Then $W^{\prime} \otimes W^{\prime \prime}=F^{0} B\left(W^{\prime}, A, W^{\prime \prime}\right) \subset F^{-1} B\left(W^{\prime}, A, W^{\prime \prime}\right) \subset \ldots$ and 
$F^{s} B\left(W^{\prime}, A, W^{\prime \prime}\right)=0$ for $s>0$. Therefore the filtration is complete. The resulting spectral sequence is known as the Eilenberg-Moore sequence. (See [61], [73].) Its $E_{1}$ term, as a chain complex, is isomorphic to $B\left(H\left(W^{\prime}\right), H(A), H\left(W^{\prime \prime}\right)\right)$, where $H(A), H\left(W^{\prime}\right), H\left(W^{\prime \prime}\right)$ are equipped with trivial differentials.

With some difference in the sign convention, our definition of $B\left(W^{\prime}, A, W^{\prime \prime}\right)$ is identical to the one used in [60].

Regard $k$ as a differential graded $A$-module, whose $A$-action is induced by the augmentation $A \rightarrow k$. Then $\bar{B}(A)=B(k, A, k)$ is known as the reduced bar construction of $A$. The bar construction of $W^{\prime \prime}$ over $A$ is $B\left(A, A, W^{\prime \prime}\right)$ [48].

If the differential $d$ of $A$ is trivial, then $B(A, A, k)$ is acyclic and is therefore the usual bar resolution.

Based on the differential formula in Proposition 1.5.1, we give a definition of $B\left(W^{\prime}, A, W^{\prime \prime}\right)$ where $A$ is a differential graded subalgebra of $\Lambda(M)$ where $A^{0}$ is not necessarily $k$.

The next theorem follows from Lemma 2.5.2.

THEOREM 4.1.1. Let $M$ be a differentiable space with $H_{0}(M)=Z$. Let $A$ be a differential graded subalgebra of $\Lambda(M)$ with $A^{0}=k$ and $A^{1} \cap d \Lambda^{0}(M)=0$. Then, for any $x_{0} \in M$, the map $B(A) \rightarrow A_{x_{0}}^{\prime}$ given by

$$
\left[w_{1}|\cdots| w_{r}\right] \mapsto \int_{x_{0}}^{x_{0}} w_{1} \cdots w_{r}
$$

is an isomorphism of differential graded algebras.

This theorem makes available methods of homological algebra for computing $H\left(A_{x_{0}}^{\prime}\right)$.

ExAmple 4.1.1. Consider the case of $M$ and $A$ satisfying the hypotheses of the above theorem such that $A$ has a trivial differential and is isomorphic to a graded polynomial algebra on $n$ generators of respective even degrees $e_{1}, \ldots, e_{n}$. By using a Koszul resolution, we conclude that

$$
H\left(A_{x_{0}}^{\prime}\right) \approx H(\bar{B}(A))=\operatorname{Tor}_{A}(k, k)
$$

which is isomorphic to an exterior algebra on $n$ generators of respective odd degrees $e_{1}-1, \ldots, e_{n}-1$. (See Theorem 2.2, Chapter VII, [48].)

4.2. The free path "fibration". The terms "fibration" and "pullback" will not be defined in generality. The differentiable map $\left(p_{0}, p_{1}\right): P(M) \rightarrow M \times$ $M$ given by $\gamma \mapsto(\gamma(0), \gamma(1))$ will be referred to as the free path fibration of the differentiable space $M$. Let $N$ be also a differentiable space. For every differentiable map $f: N \rightarrow M \times M$, there is a "pullback" diagram

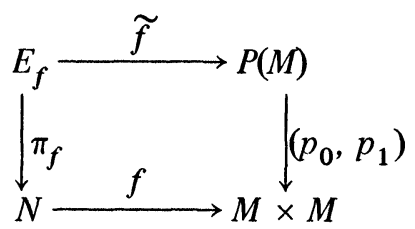

such that (a) $E_{f}$ is the differentiable subspace of $N \times P(M)$ consisting of all 
$(y, \gamma)$ such that $f y=(\gamma(0), \gamma(1))$; (b) $\pi_{f}$ is given by $(y, \gamma) \mapsto y$; and (c) $\tilde{f}$ is given by $(y, \gamma) \mapsto \gamma$. Thus $\phi: U \rightarrow E_{f}$ is a plot if and only if both $\pi_{f} \circ \phi$ and $\tilde{f} \circ \phi$ are plots.

For every differential graded subalgebra $A$ of $\Lambda(M)$, write $A_{f}^{\prime}=\pi_{f}^{*} \Lambda(N) \wedge$ $\tilde{f}^{*} A^{\prime}$ which is a subcomplex of $\Lambda\left(E_{f}\right)$.

REMARK. Let $W$ be a subcomplex of $\Lambda(N)$ such that $f^{*} \rho_{i}^{*} A \subset W, i=0,1$, where $\rho_{i}: M \times M \rightarrow M, i=0,1$, are the two projections. Then

$$
\tilde{f}^{*} p_{i}^{*} A=\pi_{f}^{*} f^{*} \rho_{i}^{*} A \subset \pi_{f}^{*} W, \quad i=0,1 .
$$

Sometimes, it will be convenient to consider the more general notion of $(A, W)_{f}^{\prime}=\pi_{f}^{*} W \wedge \tilde{f}^{*} A^{\prime}$.

For $s \geqslant 0$, define $A_{f}^{\prime}(-s)$ to be the subcomplex of $A_{f}^{\prime}$ spanned by all $\pi_{f}^{*} v \wedge \tilde{f}^{*} \int w_{1} \cdots w_{r}, w_{1}, \ldots, w_{r} \in A, r \leqslant s, v \in \Lambda(N)$. Set $A_{f}^{\prime}(s)=0$ when $s>0$. Then $\left\{A_{f}^{\prime}(s)\right\}$ is a complete descending filtration of $A_{f}^{\prime}$.

We shall attempt to determine the algebraic structure of the cochain complex $A_{f}^{\prime}$.

Equip the tensor product $\Lambda(N) \otimes \bar{B}(A)$ with a differential $d$ such that

$$
\begin{aligned}
d\left(v \otimes\left[w_{1}|\cdots| w_{r}\right]\right)= & d v \otimes\left[w_{1}|\cdots| w_{r}\right]+J v \otimes d_{\bar{B}}\left[w_{1}|\cdots| w_{r}\right] \\
& -J v \wedge \tilde{f}^{*} \rho_{0}^{*} w_{1} \otimes\left[w_{2}|\cdot \cdots| w_{r}\right] \\
& +(-1)^{v} J v \wedge \tilde{f}^{*} \rho_{1}^{*} w_{r} \otimes J\left[w_{1}|\cdots| w_{r-1}\right]
\end{aligned}
$$

where $d_{\bar{B}}$ denotes the differential of the reduced bar construction and

$$
\nu=\left(\operatorname{deg} w_{r}\right)\left(\operatorname{deg}\left[w_{1}|\cdots| w_{r-1}\right]\right) \text {. }
$$

We do not verify that $d d=0$. For cases of our interest, this will be a consequence of the next theorem.

THEOREM 4.2.1. Let $M$ be a differentiable space with $H_{0}(M)=Z$. Let $A$ be a differential graded subalgebra of $\Lambda(M)$ such that $A^{0}=k$ and $A^{1} \cap d \Lambda^{0}(M)=$ 0 . Then there is an isomorphism of cochain complexes

$$
\Lambda(N) \otimes \bar{B}(A) \approx A_{f}^{\prime}
$$

given by $v \otimes\left[w_{1}|\cdots| w_{r}\right] \mapsto \pi_{f}^{*} v \wedge \tilde{f}^{*} \int w_{1} \cdots w_{r}$.

Proof. The formula (4.2.2) is designed so that the map (4.2.3) commutes with the differential. If $\Lambda(N) \otimes \bar{B}(A)$ is equipped with the filtration $\{\Lambda(N)$ $\left.\otimes F^{s} B(k, A, k)\right\}$, then the map (4.2.3) also respects the filtration. It suffices to show that the associated graded map is an isomorphism, or, equivalently, that, for $s \geqslant 1$, the map

$$
\Lambda(N) \otimes B^{s}(k, A, k) \rightarrow A_{f}^{\prime}(-s) / A_{f}^{\prime}(-s+1)
$$

given by $v \otimes\left[w_{1}|\cdots| w_{s}\right] \mapsto \pi_{f}^{*} v \wedge \tilde{f}^{*} \int w_{1} \cdots w_{s}+A_{f}^{\prime}(-s+1)$ is an isomorphism.

Since the map (4.2.4) is evidently surjective, we are going to establish its injectiveness. Let $y_{0} \in N$ and $f y_{0}=\left(x_{0}, x_{1}\right)$. We claim that, given any $p$-simplex $\sigma: \Delta^{p} \rightarrow N$ and any compact plot chain $c$ of $P\left(M ; x_{0}, x_{1}\right)$ on $U$ strongly of order $s$, there exists a compact plot chain $\bar{c}$ of $E_{f}$ such that 


$$
\left\langle\pi_{f}^{*} v \wedge \tilde{f}^{*} \int w_{1} \cdots w_{r}, \bar{c}\right\rangle= \begin{cases}0 & \text { when } r<s \\ \langle v, \sigma\rangle\left\langle\int w_{1} \cdots w_{s}, c\right\rangle & \text { when } r=s\end{cases}
$$

(See Definition 1.6.3.)

There are sufficiently many $p$-simplices of the differentiable space $N$ to separate elements of $\Lambda^{p}(N)$ via integration, and there are sufficiently many compact plot chains of $P\left(M ; x_{0}, x_{1}\right)$ strongly of order $s$ to separate elements of $B^{s}(k, A, k)$ owing to Lemma 2.2.3. Therefore it remains to verify the mentioned claim, which implies that the map (4.2.4) is an injection.

By reparametrizing the plot chain $c$ if necessary, we may assume that $c$ is a linear combination of plots of $P\left(M ; x_{0}, x_{1}\right)$, each of which starts and ends smoothly. Construct a $p$-simplex $\tilde{\sigma}$ of $P(N)$ which ends smoothly such that $\tilde{\sigma}(\xi)(0)=\sigma(\xi)$ and $\tilde{\sigma}(\xi)(1)=y_{0}, \forall \xi \in \Delta^{p}$. Let $\tilde{\sigma}_{0}$ and $\tilde{\sigma}_{1}$ be $p$-simplices of $P(M)$ such that, for $\xi \in \Delta^{p}$ and $t \in I$,

$$
(\tilde{f} \tilde{\sigma}(\xi))(t)=\left(\tilde{\sigma}_{0}(\xi)(t), \tilde{\sigma}_{1}(\xi)(1-t)\right) .
$$

Let $\pi: \Delta^{p} \times U \rightarrow \Delta^{p}$ denote the projection. Then both $\tilde{\sigma}_{0} \times c$ and $\tilde{\sigma}_{1} \circ \pi$ are well-defined plot chains on $\Delta^{p} \times U$. Furthermore $c^{\prime}=\left(\tilde{\sigma}_{0} \times c\right) \cdot\left(\tilde{\sigma}_{1} \circ \pi\right)$ is a well-defined plot chain on $\Delta^{p} \times U$. (See §1.6.) Observe that $\bar{c}=\left(\sigma \circ \pi, c^{\prime}\right)$ is a plot chain of $E_{f} \subset N \times P(M)$ on $\Delta^{p} \times U$.

Write $u=\pi_{f}^{*} v \wedge \tilde{f}^{*} \int w_{1} \cdots w_{\mathrm{s}}$. We may assume that $\operatorname{deg} u=p+\operatorname{dim} U$. As a form on $\Delta^{p} \times U$,

$$
u_{\bar{c}}=\left(\pi_{f}^{*} v\right)_{\bar{c}} \wedge\left(\tilde{f}^{*} \int w_{1} \cdots w_{s}\right)_{\bar{c}}
$$

where $\left(\pi_{f}^{*} v\right)_{\bar{c}}=v_{\sigma} \times 1$ and, according to (1.6.1) and (1.6.2),

$$
\begin{aligned}
& \left(f^{*} \int w_{1} \cdots w_{s}\right)_{\bar{c}}=\left(\int w_{1} \cdots w_{s}\right)_{\bar{c}^{\prime}} \\
& =\sum_{0<i<j<r}\left(\left(\int w_{1} \cdots w_{i}\right)_{\tilde{\sigma}_{0}} \times\left(\int w_{i+1} \cdots w_{j}\right)\right) \wedge\left(\int w_{j+1} \cdots w_{r}\right)_{\tilde{\sigma}_{1} \circ \pi} .
\end{aligned}
$$

Since the plot chain $c$ is strongly of order $s,\left(\int w_{i+1} \cdots w_{j}\right)_{c}=0$ if $i>0$ or $j<r$ or $\operatorname{deg} w_{1} \cdots w_{s}>\operatorname{dim} U$. On the other hand, $v_{\boldsymbol{\sigma}}=0$ if $\operatorname{deg} v>p$. In summarizing, we obtain

$$
u_{\bar{c}}= \begin{cases}v_{\sigma} \times\left(\int w_{1} \cdots w_{s}\right)_{c} & \text { when } \operatorname{deg} v=p, \\ 0 & \text { when } \operatorname{deg} v \neq p .\end{cases}
$$

Repeating the above argument, we also conclude that, for $r<s$,

$$
\left(\pi_{f}^{*} v \wedge \tilde{f}^{*} \int w_{1} \cdots w_{r}\right)_{\bar{c}}=0 .
$$

Hence the theorem is proved.

REMARK. In case of $A^{0}$ containing $k$ properly, demand that $d A^{0}=A^{1} \cap$ $d \Lambda^{0}(M)$. Choose a differential graded subalgebra $\bar{A}$ of $A$ such that $\bar{A}^{0}=k$ 
and $A^{1}=\bar{A}^{1} \oplus d A^{0}$. Then the above theorem gives rise to an isomorphism

$$
\Lambda(N) \otimes \bar{B}(\bar{A}) \approx \overline{A_{f}^{\prime}}=A_{f}^{\prime} .
$$

The fact that $\overline{A_{f}^{\prime}}=A_{f}^{\prime}$ follows from formulas (4.1.2)-(4.1.4) [24]. (For this purpose, $f\left(x_{0}\right)$ and $f\left(x_{1}\right)$ in those formulas need to be replaced by $f \circ p_{0}$ and $f \circ p_{1}$ respectively.)

COROLLARY. Under the hypotheses of the theorem, the $E_{1}$ term of the spectral sequence of $A_{f}^{\prime}$ is isomorphic to $H_{D R}(N) \otimes \bar{B}(H(A))$.

4.3. A theorem on the pullback cohomology. The next assertion can be proved by using the Serre spectral sequence and Weil's work [68]. A proof in detail will appear elsewhere.

THEOREM 4.3.1. Let $M$ and $N$ be manifolds with $M$ being simply connected and $H_{*}(M)$ of finite type. Let $A$ be a differential graded subalgebra of $\Lambda(M)$ such that the inclusion $A \subset \Lambda(M)$ induces an isomorphism $H(A) \approx H_{D R}^{*}(M)$. If $E_{f}$ is the pullback of the free path fibration via a differential map $f$ : $N \rightarrow M \times M$ as in (4.2.1), then there is an isomorphism

through integration over simplices of $E_{f}$.

$$
H\left(A_{f}^{\prime}\right) \approx H^{*}\left(E_{f} ; k\right)
$$

$\mathrm{Wu}$ [72] has treated the pullback cohomology of a simplicial fibration through minimal models. Our construction of $A_{f}^{\prime}$ is analytical.

\section{APPENDIX. THE COBAR CONSTRUCTION}

Let $M$ be a differentiable space with $x_{0} \in M$. Assume that $H_{0}(M)=Z$. Recall that $\Delta(M)_{x_{0}}$ is the chain complex spanned by simplices of $M$ whose vertices are at $x_{0}$. For simplicity, write $C=\Delta(M)_{x_{0}}$.

The cobar construction $F(C)$ is the graded free associative ring generated by the set of simplices of $C$ excluding the 0 -simplex.

Let $\sigma_{1}, \ldots, \sigma_{r}$ be simplices of positive degree of $C$. Denote by $\left[\sigma_{i}\right]$ the generator of $F(C)$ corresponding to the simplex $\sigma_{i}$. We assign $\operatorname{deg}\left[\sigma_{i}\right]=-1$ $+\operatorname{deg} \sigma_{i}$ and write $\left[\sigma_{1}|\cdots| \sigma_{r}\right]=\left[\sigma_{1}\right] \cdots\left[\sigma_{r}\right]$. Set $\left[\sigma_{1}|\cdots| \sigma_{r}\right]=1$ when $r=$ 0 .

There is an augmentation $\varepsilon_{F}: F(C) \rightarrow Z$ given by $\varepsilon_{F}\left[\sigma_{1}|\cdots| \sigma_{r}\right]=1$ or 0 according as $r=0$ or $>0$.

Equip $F(C)$ with a differential $d_{F}$, which respects the multiplication such that, for any $n$-simplex $\sigma$ of $C$,

$$
d_{F}[\sigma]=[\partial \sigma]-\sum_{1<i<n}(-1)^{i}\left[{ }_{(i)} \sigma \mid \sigma_{(n-i)}\right]
$$

where ${ }_{(i)} \sigma$ and $\sigma_{(j)}$ are respectively the first $i$ - and the last $j$-faces of $\sigma$.

The purpose of this Appendix is to construct a natural chain map

$$
\mu_{F}: F(C) \rightarrow C_{*}(\Omega M)
$$

(on the category of pointed differentiable spaces), where $C_{*}$ denotes the normalized cubical chain complex. (Recall that our convention specifies degeneracy with respect to the first coordinate.) 
Theorem A4.1 is an adaptation of a theorem of Adams [1] that, for a simply connected topological space $M, \mu_{F}$ induces a homology isomorphism. The naturality of $\mu_{F}$ makes such an isomorphism differentiably meaningful for topological differentiable spaces. For example, if $M$ is a simply connected manifold, then the isomorphism

$$
\mu_{F_{*}}: H\left(F\left(\Delta(M)_{x_{0}}\right)\right) \approx H_{*}(\Omega M)
$$

becomes valid in the smooth sense.

Our construction of $\mu_{F}$ differs technically from that of Adams in order to take care of differentiability requirements and to allow possible applications to certain nonsimply connected cases. On the large, we follow Adams' original work, which is geometrical and suits the needs of this Appendix. A recent result of Dwyer [32] seems to indicate that Theorem A4.1 can be improved to include the case where $\pi_{1}(M)$ acts nilpotently on $H_{*}(\Omega M)$.

A1. Moore paths. A usual Moore path is a map $[0, a] \rightarrow M$, where $a \geqslant 0$. Moore paths possess the advantage of having an associative multiplication. In the same spirit, we define a Moore path of a differentiable space $M$ to be a pair $(\gamma, a)$, where $\gamma: I \rightarrow M$ is a (smooth) path, and $a$ is a nonnegative number which can become zero only when $\gamma$ is a constant path. Denote by $\underline{P}(M)$ the differentiable subspace of $P(M) \times R$ consisting of all Moore paths of $M$.

If $\left(\gamma^{\prime}, a^{\prime}\right)$ and $\left(\gamma^{\prime \prime}, a^{\prime \prime}\right)$ are Moore paths of $M$ with $\gamma^{\prime}(1)=\gamma^{\prime \prime}(0)$, define the product

$$
(\gamma, a)=\left(\gamma^{\prime}, a^{\prime}\right) *\left(\gamma^{\prime \prime}, a^{\prime \prime}\right)
$$

such that $a=a^{\prime}+a^{\prime \prime}$ and

$$
\gamma(t)= \begin{cases}\gamma^{\prime}\left(a t / a^{\prime}\right), & \text { for } t \in\left[0, a^{\prime} / a\right], \\ \gamma^{\prime \prime}\left(\left(a t-a^{\prime}\right) / a^{\prime \prime}\right) & \text { for } t \in\left[a^{\prime} / a, 1\right],\end{cases}
$$

provided $a^{\prime}>0$ and $a^{\prime \prime}>0$. When $a^{\prime}=0$ (resp. $a^{\prime \prime}=0$ ), define $\gamma=\gamma^{\prime \prime}$ (resp. $\left.\gamma^{\prime}\right)$. Of course, the product exists only when the map $\gamma: I \rightarrow M$ is indeed a plot of $M$.

Let $\underline{\Omega} M$ (resp. $\left.\underline{P}\left(M ;-, x_{0}\right), \underline{P}\left(M ; x_{0},-\right)\right)$ be the differentiable subspace of $\underline{P}(M)$ consisting of all Moore paths $(\gamma, a)$ with $\gamma \in \Omega M$ (resp. $P\left(M ;-, x_{0}\right)$, $\left.\bar{P}\left(M ; x_{0},-\right)\right)$.

Every plot $\underline{\alpha}: U \rightarrow \underline{P}(M)$ can be taken as a pair $(\alpha, a)$ where $\alpha: U \rightarrow P(M)$ is a plot and $a: U \rightarrow R$ is a nonnegative valued function on $U$. We say that a plot $\underline{\alpha}=(\alpha, a)$ of $P(M)$ is smoothed if $a$ is strictly positive and if the plot $\alpha$ starts and ends smoothly. (See \$2.2.)

Let $\underline{\alpha}: U \rightarrow \underline{P}(M)$ and $\underline{\alpha}^{\prime}: U^{\prime} \rightarrow \underline{P}(M)$ be smoothed plots with $\alpha(\xi)(1)=$ $x_{0}=\alpha^{\prime}\left(\xi^{\prime}\right)(0), \forall\left(\xi, \xi^{\prime}\right) \in U \times U^{\prime}$. Let $\pi$ and $\pi^{\prime}$ be projections from $U \times U^{\prime}$ to $U$ and $U^{\prime}$ respectively. Define

$$
\underline{\alpha} * \underline{\alpha}^{\prime}=\left(\beta, a+a^{\prime}\right): U \times U^{\prime} \rightarrow \underline{P}(M)
$$

by $\left(\xi, \xi^{\prime}\right) \mapsto \underline{\alpha}(\xi) * \underline{\alpha}^{\prime}\left(\xi^{\prime}\right)$. According to Corollary, Lemma 2.2.1, $\beta$ is a plot of $P(M)$ resulting from the plots $\alpha \circ \pi, \alpha^{\prime} \circ \pi^{\prime}$ and the function $a /\left(a+a^{\prime}\right)$. Moreover, the plot $\beta$ starts and ends smoothly. Thus there is an associative multiplication for the set of smoothed plots of $\underline{\Omega} M$. In particular, if $\underline{\alpha}$ and $\underline{\alpha}^{\prime}$ 
are smoothed cubes of $\underline{\Omega} M$, verify that

$$
\partial\left(\underline{\alpha} * \underline{\alpha}^{\prime}\right)=\partial \underline{\alpha} * \underline{\alpha}^{\prime}+(-1)^{\operatorname{deg} \underline{\alpha}} \alpha * \partial \underline{\alpha}^{\prime} .
$$

Write $\underline{\eta}_{x_{0}}=\left(\eta_{x_{0}}, 0\right) \in \underline{\Omega} M$. Then the 0 -cube $\left(\underline{\eta}_{x_{0}}\right)$ serves as a unit element for the multiplication $*$ of smoothed plots of $\underline{\Omega} \bar{M}$.

Let $v_{0}, \ldots, v_{n}$ be the vertices of the standard $n$-simplex $\Delta^{n}$, which is embedded in $R^{n+1}$ so that $v_{0}=(1,0, \ldots, 0), v_{1}=(0,1,0, \ldots, 0), \ldots, v_{n}=$ $(0, \ldots, 0,1)$.

In the remainder of this section, we are going to construct, inductively on $n \geqslant 1$, cubes $\theta_{n}: I^{n-1} \rightarrow P\left(\Delta^{n}: v_{0}, v_{n}\right)$, which will be used to construct the chain map $\mu_{F}$.

A cube $\phi: I^{n} \rightarrow U \subset R^{m}$ is said to be constant along normals to $i^{n}$ if, if there exists $\varepsilon>0$ such that, for any $\xi \in I^{n}, \partial \phi / \partial \xi^{i}=0$ when either $\xi^{i}<\varepsilon$ or $1-\xi^{i}<\varepsilon, 1 \leqslant i \leqslant n$.

LEMMA A1.1. Let $U$ be a convex subset of $R^{m}$. Let $\psi: i^{n+1} \rightarrow U$ be a continuous map such that its restriction to each $n$-face of $I^{n+1}$ is smooth and constant along normals to its boundary. Then $\psi$ can be extended to a smooth $\operatorname{map} \phi: I^{n+1} \rightarrow U$

Proof. First extend $\psi$ to a neighborhood $N$ of $I^{n+1}$ in $I^{n+1}$ such that $\psi$ is constant along normals to $i^{n+1}$. Choose $x_{0} \in U$ and set

$$
\phi(\xi)=(1-\chi(\xi)) \psi(\xi)+\chi(\xi) x_{0}
$$

where $\chi: I^{n+1} \rightarrow I$ is a $C^{\infty}$ function such that $\chi(\xi)=1$ about $\dot{I}^{n+1}$. This proves the lemma.

Let $f_{i}^{n}, l_{i}^{n}: \Delta^{i} \rightarrow \Delta^{n}$ be respectively the first and the last $i$-face injections and let $\partial_{i}: \Delta^{n-1} \rightarrow \Delta^{n}$ be the $i$ th face injection. Let $\lambda_{i}^{\varepsilon}: I^{n-2} \rightarrow I^{n-1}, 1 \leqslant i \leqslant n-$ $1, \varepsilon=0,1$, be the $i$ th front- and aft-face injections given by

$$
\left(\xi^{1}, \ldots, \hat{\xi}^{i}, \ldots, \xi^{n-1}\right) \mapsto\left(\xi^{1}, \ldots, \xi^{i-1}, \varepsilon, \xi^{i+1}, \ldots, \xi^{n-1}\right) .
$$

Denote by $a_{n}: P\left(\Delta^{n}\right) \rightarrow R$ the energy function given by

$$
\left.a_{n}(\gamma)=\int_{0}^{1} \sum\left[d x^{i}(\gamma(t)) / d t\right)\right]^{2} d t
$$

We embed $P\left(\Delta^{n}\right)$ in $\underline{P}\left(\Delta^{n}\right)$ by identifying the path $\gamma$ and the Moore path $\left(\gamma, a_{n}(\gamma)\right)$. In this sense, the multiplication $*$ makes sense for plots of $P\left(\Delta^{n}\right)$.

Choose a $C^{\infty}$ function $\zeta: I \rightarrow I$ such that $\zeta(t)=0$ (resp. 1) for $t$ sufficiently close to 0 (resp. 1).

LEMMA A1.2. For $n \geqslant 1$, there exist cubes $\theta_{n}: I^{n-1} \rightarrow P\left(\Delta^{n} ; v_{0}, v_{n}\right)$ satisfying the following conditions:

(a) For $1 \leqslant i \leqslant n-1$,

$$
\theta_{n} \circ \lambda_{i}^{0}=P\left(\partial_{i}\right) \theta_{n-1} \text { and } \theta_{n} \circ \lambda_{i}^{1}=P\left(f_{i}^{n}\right) \theta_{i} * P\left(l_{n-i}^{n}\right) \theta_{n-1} .
$$

(b) The $\Delta^{n}$-valued function $\phi_{\theta_{n}}$ is constant along normals to $i^{n}$.

Proof. Let $\theta_{1}$ be given by

$$
\theta(0)(t)=(1-\zeta(t)) v_{0}+\zeta(t) v_{1} .
$$


For $n>1$, we need to construct $\phi_{\theta_{n}}: I^{n}=I \times I^{n-1} \rightarrow \Delta^{n}$ whose restrictions to the $(n-1)$-faces are determined inductively by the following requirements:

(i) $\phi_{\theta_{n}}(0, \xi)=v_{0}$ and $\phi_{\theta_{n}}(1, \xi)=v_{n}$ for $\xi \in I^{n-1}$.

(ii) For $1 \leqslant i \leqslant n-1$, and

$\phi_{\theta_{n}}\left(t, \xi^{1}, \ldots, \xi^{i-1}, 0, \xi^{i+1}, \ldots, \xi^{n-1}\right)=\partial_{i}\left(\theta_{n-1}\left(\xi^{1}, \ldots, \hat{\xi}^{i}, \ldots, \xi^{n-1}\right)(t)\right)$

$$
\begin{aligned}
\phi_{\theta_{n}}\left(t, \xi^{1}, \ldots, \xi^{i-1}, 1, \xi^{i+1}, \ldots, \xi^{n-1}\right) \\
\quad=\left(P\left(f_{i}^{n}\right) \theta_{i}\left(\xi^{1}, \ldots, \xi^{i-1}\right) * P\left(l_{n-i}^{n}\right) \theta_{n-i}\left(\xi^{i+1}, \ldots, \xi^{n-1}\right)\right)(t) .
\end{aligned}
$$

Verify that these requirements are consistent on the $(n-2)$-faces and that the restriction of $\phi_{\theta_{n}}$ to each $(n-1)$-face is constant along normals to its boundary. Hence we may apply Lemma A1.1 to complete the proof.

Observe that $l_{n-1}^{n}=\partial_{0}$ and $f_{n-1}^{n}=\partial_{n}$ so that, for $n \geqslant 2$,

$$
\begin{aligned}
\partial \theta_{n}= & \sum(-1)^{i}\left(\lambda_{i}^{0} \theta_{n}-\lambda_{i}^{1} \theta_{n}\right) \\
= & \sum_{0<i<n}(-1)^{i} P\left(\partial_{i}\right) \theta_{n-1}-P\left(l_{n-1}^{n}\right) \theta_{n-1}-(-1)^{n} P\left(f_{n-1}^{n}\right) \theta_{n-1} \\
& -\sum_{0<i<n}(-1)^{i} P\left(f_{i}^{n}\right) \theta_{i} * P\left(l_{n-i}^{n}\right) \theta_{n-i} \cdot
\end{aligned}
$$

LeMma A1.3. For every $n$-form $w$ on $\Delta^{n}$,

$$
\int_{\phi_{\theta_{n}}} w=\int_{\Delta^{n}} w
$$

Proof. Write $w=d u, u$ being an $(n-1)$-form on $\Delta^{n}$. Observe that $\lambda_{1}^{\varepsilon} \phi_{\theta_{n}}$, $\varepsilon=0,1$, are constant cubes and that the image of each $\lambda_{i+1}^{1} \phi_{\theta_{n}}=\phi_{\theta_{n} \circ \lambda_{i}^{\prime}}$, $1<i<n-1$, lies in the $(n-2)$-skeleton of $I^{n}$. Hence

$$
\begin{aligned}
\int_{\phi_{\theta_{n}}} w & =\int_{\partial \phi_{\theta_{n}}} u=\sum_{1 \leqslant i \leqslant n-1}(-1)^{i} \int_{\partial_{i} \circ \phi_{\theta_{n}}} u+\int_{\phi_{\lambda \mid \theta_{n}}} u+(-1)^{n} \int_{\phi_{\lambda_{h}^{\prime}-\theta_{n}}} u \\
& =\int_{\partial \Delta^{n}} u=\int_{\Delta^{n}} w .
\end{aligned}
$$

COROLlary. As a continuous map of pairs,

$$
\phi_{\theta_{n}}:\left(I^{n}, \dot{I}^{n}\right) \rightarrow\left(\Delta^{n}, \dot{\Delta}^{n}\right)
$$

is of degree 1.

A2. The natural chain map $\mu_{F}$. For every $n$-simplex $\sigma: \Delta^{n} \rightarrow M, n \geqslant 1$, denote by $\hat{\sigma}$ the $(n-1)$-cube

$$
I^{n-1} \stackrel{\theta_{n}}{\rightarrow} P\left(\Delta^{n}\right) \stackrel{P(\sigma)}{\rightarrow} P(M) .
$$

Then, for any $n$-form $w$ on $M,\left\langle\int w, \hat{\sigma}\right\rangle=\int_{a} w$.

Denote by $\underline{\sigma}$ the smoothed $(n-1)$-cube $\left(\hat{\sigma}, a_{n} \circ \theta_{n}\right)$ of $\underline{P}(M)$. It follows from (A1.2) that 


$$
\begin{aligned}
\partial \underline{\sigma}= & \sum_{0<i<n}(-1)^{i} \sigma^{(i)}-\underline{\sigma_{(n-1)}}-(-1)^{n} \underline{(n-1)} \sigma \\
& -\sum_{0<i<n}(-1)^{i} \underline{(i)} \sigma \underline{\sigma_{(n-i)}}
\end{aligned}
$$

where $\sigma^{(i)}=\partial_{i} \circ \sigma$ denotes the $i$ th face of $\sigma$.

Let $C_{*}^{\prime}(\Omega M)$ be the (unnormalized) cubical chain complex of the Moore loop space $\underline{\Omega} M$. Define a graded ring homomorphism

$$
\tilde{\mu}_{F}: F(C) \rightarrow C_{*}^{\prime}(\underline{\Omega} M)
$$

such that $\tilde{\mu}_{F}[\sigma]=\underline{\sigma}-\left(\underline{\eta}_{x_{0}}\right)$ or $\underline{\sigma}$ according as $\operatorname{deg} \sigma=1$ or $>1$. Recall that $\left(\underline{\eta}_{x_{0}}\right)$ serves as the unit element for the multiplication * of $C_{*}^{\prime}(\underline{\Omega} M)$. Denote by $\mu_{F}$ the composite map

$$
F(C) \stackrel{\tilde{\mu}_{F}}{\rightarrow} C_{*}^{\prime}(\underline{\Omega} M) \rightarrow C_{*}(\Omega M)
$$

where the second arrow is induced by the forgetful map $\Omega M \rightarrow \Omega M$. Then $\mu_{F}$ is natural on the category of pointed differentiable spaces. The formula (A2.1) implies that

$$
\partial \tilde{\mu}_{F}[\sigma]=\tilde{\mu}_{F} d_{F}[\sigma]
$$

so that $\mu_{F}$ is indeed a chain map.

We shall be interested in the case of $M$ being a topological differentiable space with an underlying topological space ${ }_{r} M$ such that the inclusion $\Delta(M)_{x_{0}} \subset \Delta\left({ }_{T} M\right)_{x_{0}}$ induces homology isomorphism. There are two chain maps $\mu_{F}(M)$ and $\mu_{F}\left({ }_{T} M\right)$, which give rise to a commutative diagram

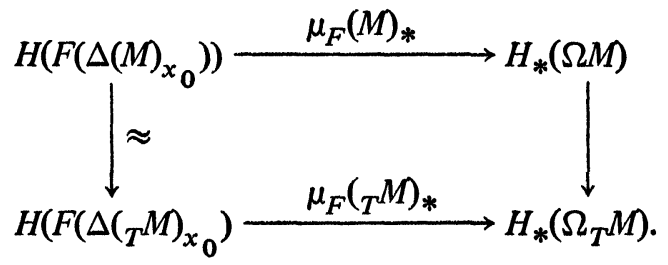

If $\mu_{F}\left({ }_{T} M\right)_{*}$ is an isomorphism, then $\mu_{F}(M)_{*}$ is only a monomorphism. If, moreover, $H_{*}(\Omega M) \approx H_{*}\left(\Omega_{T} M\right)$, then $\mu_{F}(M)_{*}$ becomes an isomorphism.

For a topological differentiable space $M$, there are three loop spaces at a given base point $x_{0}$, namely,

(a) the differentiable loop space $\Omega M$;

(b) the underlying topological space ${ }_{T} \Omega M$ with compact open topology;

(c) the continuous loop space $\Omega_{T} M$.

In the case of $M$ being a differentiable manifold, there are canonical isomorphisms

$$
H_{*}(\Omega M) \approx H_{*}\left(T^{\Omega M}\right) \approx H_{*}\left(\Omega_{T} M\right)
$$

The first isomorphism can be obtained by applying Lemma 1.3.1, and the second isomorphism follows from the known result that the inclusion $T^{\Omega M \subset}$ $\Omega_{T} M$ is a homotopy equivalence. (This can be proved by using the proof of Theorem 17.1 [53].) 
A3. The acyclic twisted tensor product $F(C) \otimes_{Z} C$. The remainder of this Appendix is devoted to a proof of Theorem A4.1. Hereafter, $M$ will denote a topological space. Then $C=\Delta(M)_{x_{0}}$ is the usual singular chain complex with all vertices at the base point $x_{0}$. We are going to equip $F(C) \otimes_{Z} C$ with a differential $d_{L}$ so that the chain complex is acyclic. Our main effort in this section is to extend the natural chain map $\mu_{F}$ to a natural chain map

$$
\mu_{L}: F(C) \otimes_{Z} C \rightarrow C_{*}(P M)
$$

where $P M=P\left(M ; x_{0},-\right)$.

Let $\sigma, \sigma_{1}, \ldots, \sigma_{r}$ be simplices of $C$, where $\sigma_{1}, \ldots, \sigma_{r}$ are of positive degree. Then $F(C) \otimes_{Z} C$ has, as a basis, all elements of the type

$$
\left[\sigma_{1}|\cdot \cdots| \sigma_{r}\right] \sigma=\left[\sigma_{1}|\cdots| \sigma_{r}\right] \otimes_{Z} \sigma
$$

whose degree is equal to $\operatorname{deg}\left[\sigma_{1}|\cdots| \sigma_{r}\right]+\operatorname{deg} \sigma$.

Define the differential $d_{L}$ so that

$$
d_{L}\left(u \otimes_{Z} \sigma\right)=\left(d_{F} u\right) \otimes_{Z} \sigma+(-1)^{\operatorname{deg} u} u d_{L}([] \sigma),
$$

and, when $n=\operatorname{deg} \sigma>0$,

$$
d_{L}[] \sigma=[] \partial \sigma-\sum_{0<i \leqslant n}(-1)^{i}\left[{ }_{(i)} \sigma\right] \sigma_{(n-i)} .
$$

When $n=0$, we have $\sigma=\left(x_{0}\right)$ and $d_{L}[]\left(x_{0}\right)=0$. Define the graded map of degree 1

$$
s_{L}: F(C) \otimes_{Z} C \rightarrow F(C) \otimes_{Z} C
$$

such that $s_{L}\left(\left[\sigma_{1}|\cdots| \sigma_{r}\right] \sigma\right)=0$ or $\left[\sigma_{1}|\cdots| \sigma_{r-1}\right] \sigma_{r}$ according as $n>0$ or $=0$. Verify that $d_{1} s_{L}+s_{L} d_{L}=1-\varepsilon_{L}$ and $d_{L} d_{L}=0$, where $\varepsilon_{L}$ is the obvious augmentation of $F(C) \otimes_{Z} C$. Therefore the chain complex $F(C) \otimes_{Z} C$ is acyclic.

Write $\underline{P} M=\underline{P}\left(M ; x_{0},-\right)$. It will follow from Lemma A3.1 that, for every $n$-simplex $\sigma$ of $C$, we can construct an $n$-cube

$$
\underline{c}_{\sigma}: I^{n} \rightarrow \underline{P} M
$$

such that, when $n=0, \underline{c}_{\sigma}=\left(\underline{\eta}_{x_{0}}\right)$, and, when $n>0$,

$$
\partial \underline{c}_{\sigma}=\sum_{0 \leqslant i \leqslant n}(-1)^{i} \underline{\mathcal{c}}_{\sigma^{\prime \prime \prime}}-\sum_{0<i \leqslant n}(-1)^{i} \tilde{\mu}_{F}\left({ }_{(i)} \sigma\right) * \underline{c}_{\sigma_{\mid n},{ }^{\prime}}
$$

Define $\tilde{\mu}_{L}: F(C) \otimes_{Z} C \rightarrow C_{*}^{\prime}(\underline{P} M)$ such that

$$
\tilde{\mu}_{L}\left(\left[\sigma_{1}|\cdots| \sigma_{r}\right] \sigma\right)=\tilde{\mu}_{F}\left[\sigma_{1}|\cdots| \sigma_{r}\right] * \underline{c}_{\sigma} .
$$

Then (A3.1) becomes $\partial \tilde{\mu}_{L}([] \sigma)=\tilde{\mu}_{L} d_{L}[] \sigma$. It follows from (A1.1) that $\tilde{\mu}_{L}$ is a chain map. Define $\mu_{L}$ to be the composite map

$$
F(C) \otimes_{Z} C \stackrel{\tilde{\mu}_{L}}{\rightarrow} C_{*}^{\prime}(\underline{P} M) \stackrel{\text { canonical }}{\rightarrow} C_{*}(P M) .
$$

Let $P\left(\Delta^{n} ; v_{0},-\right)$ be given the compact open topology. For any $\gamma^{\prime}, \gamma^{\prime \prime} \in$ $P\left(\Delta^{n} ; v_{0},-\right)$ and $0 \leqslant s \leqslant 1$, define

$$
\gamma_{s}=(1-s) \gamma^{\prime}+s \gamma^{\prime \prime} \in P\left(\Delta^{n} ; v_{0},-\right)
$$


such that $\gamma_{s}(t)=(1-s) \gamma^{\prime}(t)+s \gamma^{\prime \prime}(t)$. Then the arc length $a_{n}\left(\gamma_{s}\right)$ depends continuously on $s$.

LEMMA A3.1. There exists a family of continuous maps

$$
\tau_{n}: I^{n} \rightarrow P\left(\Delta^{n} ; v_{0},-\right), \quad n \geqslant 0,
$$

such that, for $(t, \xi)$ in a neighborhood of $\{0\} \times I^{n}$,

$$
\tau_{n}(\xi)(t)=v_{0}
$$

and, for $1 \leqslant i \leqslant n$,

$$
\begin{aligned}
\tau_{n} \circ \lambda_{i}^{0} & =P\left(\partial_{i}\right) \tau_{n-1} ; \\
\tau_{n} \circ \lambda_{i}^{1} & =P\left(f_{i}^{n}\right) \theta_{i} * P\left(l_{n-i}^{n}\right) \tau_{n-i},
\end{aligned}
$$

where $\theta_{1}, \theta_{2}, \ldots$ are as defined in $\S \mathrm{Al}$.

Proof. The map $\tau_{0}$ is uniquely defined. For $n \geqslant 1$, the condition (A3.2) ensures that $\tau_{n} \circ \lambda_{i}^{1}$ are well-defined maps into $P\left(\Delta^{n} ; v_{0},-\right)$.

Verify that the conditions (A3.3) are consistent on the $(n-2)$-skeleton of $I^{n}$ so that the restriction $\tau_{n} \mid \dot{I}^{n}$ is well defined through the induction hypothesis. Choose $\gamma \in P\left(\Delta^{n} ; v_{0},-\right)$ and $\xi_{0} \in I^{n}-I^{n}$. Define $\tau_{n}$ such that, for any $\xi \in i^{n}$,

$$
\tau_{n}\left((1-s) \xi_{0}+s \xi\right)=(1-s) \gamma+s \tau_{n}(\xi) .
$$

Then $a_{n} \circ \tau_{n}$ is continuous. Hence the lemma is proved.

If $\sigma: \Delta^{n} \rightarrow M$ is an $n$-simplex, define $c_{\sigma}=P(\sigma) \tau_{n}$ and $\underline{c}_{\sigma}=\left(c_{\sigma}, a_{n} \circ \tau_{n}\right)$. Then (A3.1) follows.

REMARK. Since every vertex of $\tau_{n}$ must be a path from $v_{0}$ to some vertex of $\Delta^{n}$, each vertex of the cube $c_{\sigma}$ must lie in $\Omega M$.

A4. The Adams theorem. Our adaption of the main theorem of Adams [1] reads as follows:

THEOREM A4.1. If $M$ is a simply connected topological space with a base point $x_{0}$, then the chain map

$$
\mu_{F}: F\left(\Delta(M)_{x_{0}}\right) \rightarrow C_{*}(\Omega M)
$$

induces an isomorphism

$$
H\left(F\left(\Delta(M)_{x_{0}}\right)\right) \approx H_{*}(\Omega M) .
$$

Proof. For this proof, $C_{*}(P M)$ will denote the subcomplex of the normalized cubical chain complex of $P M$ spanned by those nondegenerate cubes whose vertices are in $\Omega M$. Then $C_{*}(P M)$ is acyclic and has the usual filtration $F$ such that $F_{p} C_{*}(P M)$ is the subcomplex spanned by all nondegenerate $n$-cubes $\alpha$ of $P M$ such that either $n \leqslant p$ or $\alpha\left(\xi^{1}, \ldots, \xi^{n}\right)(1)$ depends only on the last $p$ coordinates. This filtration gives rise to a Serre spectral sequence $\left\{S^{r}, d^{r}\right\}_{r \geqslant 0}$ with

$$
S_{p, q}^{1} \approx H_{q}(\Omega M) \otimes_{Z} C_{p} \quad\left(\text { where } C=\Delta(M)_{x_{0}}\right)
$$

and

$$
S_{p, q}^{2} \approx H_{p+q}\left(M ; H_{q}(\Omega M)\right) .
$$


(See [59] or [41].)

On the other hand, the chain complex $F(C) \otimes_{Z} C$ also has a filtration $F$ such that $F_{p}\left(F(C) \otimes_{Z} C\right)$ is the subcomplex spanned by all $\left[\sigma_{1}|\cdots| \sigma_{r}\right] \sigma$, $r \geqslant 0$, with $\operatorname{deg} \sigma \leqslant p$. Let $\left\{\bar{S}^{r}, \bar{d}^{r}\right\}_{r \geqslant 0}$ denote the resulting spectral sequence.

Verify, in a standard manner, the isomorphism

$$
\bar{S}_{p, q}^{2} \approx H_{p+q}\left(C ; H_{q}(F(C))\right), \quad p, q \geqslant 0 .
$$

Owing to a remark in the last section, the natural chain map $\mu_{L}: F(C)$ $\otimes_{Z} C \rightarrow C_{*}(P M)$ is well defined with $C_{*}(P M)$ being spanned by cubes with vertices in $\Omega M$. We assert that $\mu_{L}$ respects the filtrations. In fact, let $\rho$ : $C_{*}^{\prime}(\underline{P} M) \rightarrow C_{*}(P M)$ denote the canonical chain map. Then for any $p$-simplex $\sigma$ and simplices $\sigma_{1}, \ldots, \sigma_{r}$ of positive degree in $C$,

$$
\mu_{L}\left(\left[\sigma_{1}|\cdots| \sigma_{r}\right] \sigma\right)=\rho\left(\tilde{\mu}_{F}\left[\sigma_{1}|\cdots| \sigma_{r}\right] * \underline{c}_{\sigma}\right)
$$

is a linear combination of $(n+p)$-cubes $\alpha, n=\operatorname{deg}\left[\sigma_{1}|\cdots| \sigma_{r}\right]$, such that

$$
\alpha\left(\xi^{1}, \ldots, \xi^{n}, \eta^{1}, \ldots, \eta^{p}\right)(1)=c_{\sigma}\left(\eta^{1}, \ldots, \eta^{p}\right)(1) .
$$

Therefore $\mu_{L}\left(F_{p}\left(F(C) \otimes_{Z} C\right)\right) \subset F_{p} C_{*}(P M)$.

The chain map $\mu_{L}$ induces spectral sequence homomorphisms. Among these homomorphisms, there are homomorphisms

$$
H_{p}(C) \approx \bar{S}_{p, o}^{2} \rightarrow S_{p, o}^{2} \approx H_{p}(M), \quad p \geqslant 0,
$$

which are induced by the natural chain map

$$
\nu: C=\Delta(M)_{x_{0}} \rightarrow C_{*}(M)
$$

given by $\sigma \mapsto p_{1} * \mu_{L}$ ([ ] ]). (Recall that $p_{1}: P M \rightarrow M$ is given by $\gamma \mapsto \gamma(1)$.) The method of acyclic models can be used to establish the maps (A4.2) are isomorphisms.

Now both spectral sequences $\left\{S^{r}\right\}$ and $\left\{\bar{S}^{r}\right\}$ converge. Since both chain complexes $C_{*}(P M)$ and $F(C) \otimes_{Z} C$ are acyclic, $\mu_{L}$ induces trivial isomorphisms

$$
\bar{S}_{p, q}^{\infty} \approx S_{p, q}^{\infty}, \quad p, q \geqslant 0 .
$$

Appealing to the comparison theorem (see, e.g. Theorem 11.1 [48]), we obtain the isomorphisms

$$
H_{q}(F(C)) \approx \bar{S}_{0, q}^{2} \underset{\rightarrow}{\stackrel{\sim}{\rightarrow}} S_{0, q}^{2} \approx H_{q}(\Omega M), \quad q>0 .
$$

\section{BIBLIOGRAPHY}

1. J. F. Adams, On the cobar construction, Colloque de topologie algébrique (Louvain, 1956), George Thone, Liège; Masson, Paris, 1957, pp. 81-87. MR 19, 759.

2. J. F. Adams and P. J. Hilton, On the chain algebra of a loop space, Comment. Math. Helv. 30 (1956), 305-330. MR 17, 1119.

3. L. V. Ahlfors and L. Sario, Riemann surfaces, Princeton Univ. Press, Princeton, N. J., 1960. MR 22 \#5729.

4. S. I. Al'ber, The topology of functional manifolds and the calculus of variation in the large, Uspehi Mat. Nauk 25 (154) (1970), no. 4, 57-122 = Russian Math. Surveys 25 (1970), no. 4, 51-117. MR 43 \#553. 
5. A. Asada, Algebraic cohomology of loop spaces, J. Fac. Sci. Shinshu Univ. 4 (1969), 1-23. MR 41 \#9241.

6. _ Representations of loop spaces and fibre bundles, J. Fac. Sci. Shinshu Univ. 4 (1969), 39-56. MR 41 \# 9256.

7. G. Baumslag, On the residual finiteness of generalized free products of nilpotent groups, Trans. Amer. Math. Soc. 106 (1963), 193-209. MR 26 \#2489.

8. R. Bott, The space of loops on a Lie group, Michigan Math. J. 5 (1958), 35-61. MR 21 \#1589.

9. R. Bott and H. Samelson, On the Pontryagin product in spaces of paths, Comment. Math. Helv. 27 (1953), 320-337 (1954). MR 15, 643.

10. __ Applications of the theory of Morse to symmetric spaces, Amer. J. Math. 80 (1958), 964-1029. MR 21 \#4430.

11. E. H. Brown, Jr., Twisted tensor products. I, Ann. of Math. (2) 69 (1959), 223-246. MR 21 \#4423.

12. H. Cartan, Sur les groupes d'Eilenberg-Mac Lane $H(\Pi, n)$. I. Méthode des contructions, Proc. Nat. Acad. Sci. U. S. A. 40 (1954), 467-471. MR 16, 390.

13. K. -T. Chen, Iterated integrals and exponential homomorphisms, Proc. London Math. Soc. (3) 4 (1954), 502-512. MR 17, 394.

14. _ Integration of paths, geometric invariants and a generalized Baker-Hausdorff formula, Ann. of Math. (2) 65 (1957), 163-178. MR 19, 12.

15. Integration of paths-a faithful representation of paths by non-commutative formal power series, Trans. Amer. Math. Soc. 89 (1958), 395-407. MR 21 \#492.

16. __ Formal differential equations, Ann. of Math. (2) 73 (1961), 110-133. MR 27 \# 371.

17. _ Iterated path integrals and generalized paths, Bull. Amer. Math. Soc. 73 (1967), 935-938. MR 36 \#806.

18. _ Algebraic paths, J. Algebra 10 (1968), 8-36. MR 37 \# 5204.

19. __ Covering-space-like algebras, J. Algebra 13 (1969), 308-326. MR 41 \# 8403.

20. __ An algebraic dualization of fundamental groups, Bull. Amer. Math. Soc. 75 (1969), 1020-1024. MR 41 \#5455.

21. Algebras of iterated path integrals and fundamental groups, Trans. Amer. Math. Soc. 156 (1971), 359-379. MR 43 \# 1069.

22. ___ Differential forms and homotopy groups, J. Differential Geometry 6 (1971), 231-246.

23. ___ Free subalgebras of loop space homology and Massey products, Topology 11 (1972), 237-243. MR 45 \#4411.

24. Iterated integrals of differential forms and loop space homology, Ann. of Math. (2) 97 (1973), 217-246.

25. __ Fundamental groups, nilmanifolds and iterated integrals, Bull. Amer. Math. Soc. 79 (1973), 1033-1035. MR 47 \#7676.

26. Iterated integrals, fundamental groups and covering spaces, Trans. Amer. Math. Soc. 206 (1975), 83-98.

27. Connections, holonomy and path space homology, Differential Geometry (Proc. Sympos. Pure Math., vol 27), Part 1, Amer. Math. Soc., Providence, R. I., 1975, pp. 39-52.

28. Solvability on manifolds by quadratures permitting only integrals, Bull. Amer. Math. Soc. 80 (1974), 1210-1212. MR 50 \#5845.

29. , Algebra, Topology and Category Theory, A Collection of Papers in Honor of Samuel

Eilenberg, Academic Press, New York 1976, 19-32.

30. Extension of $C^{\infty}$ function algebra by integrals and Malcev completion of $\pi_{1}$, Advances in Math. 23 (1977), 181-210.

31. P. Deligne, P. Griffiths, J. Morgan and D. Sullivan, Real homotopy theory of Kähler manifolds, Invent. Math. 29 (1975), 245-274.

32. W. G. Dwyer, Strong convergence of the Eilenberg-Moore spectral sequence, Topology 13 (1974), 255-265.

33. S. Eilenberg, Singular homology in differentiable manifolds, Ann. of Math. (2) 48 (1947), 670-681. MR 9, 52.

34. S. Eilenberg and S. Mac Lane, On the groups of $H(\pi, n)$. I, Ann. of Math. (2) 58 (1953), 55-106. MR 15, 54.

35. S. Eilenberg and J. C. Moore, Homology and fibrations. I, Comment. Math. Helv. 40 (1966), 199-236. MR 34 \# 3579. 
36. S. Eilenberg and J. C. Moore, Limits and spectral sequences, Topology 1 (1962), 1-23. MR 26 \#6229.

37. E. Friedlander, P. A. Griffiths and J. Morgan, Homotopy theory and differential forms, Seminario di Geometria, Firenz (1972), mimeographed.

38. T. Ganea, On the loop spaces of projective spaces, J. Math. Mech. 16 (1967), 853-858. MR 34 \#6766.

39. V. K. A. M. Gugenheim, On the chain-complex of a fibration, Illinois J. Math. 16 (1972), 398-414. MR 46 \#891.

40. P. J. Hilton and S. Wylie, Homology theory, Cambridge Univ. Press, New York, 1960. MR 22 \# 5963.

41. S. -T. Hu, Homotopy theory, Academic Press, New York and London, 1959. MR 21 \#5186.

42. H. H. Johnson, A generalization of K.-T. Chen's invariants for paths under transformation groups, Trans. Amer. Math. Soc. 105 (1962), 453-461. MR 25 \# 5125.

43. W. Klingenberg, Mimeographed notes on closed geodesics (to appear in book form).

44. S. Kobayashi and K. Nomizu, Foundations of differential geometry. Vol. I, Interscience, New York, 1963. MR 27 \#2945.

45. E. R. Kolchin, Differential algebra and algebraic groups, Academic Press, New York, 1973.

46. D. Kraines, Massey higher products, Trans. Amer. Math. Soc. 124 (1966), 431-449. MR 34 \#2010.

47. __ Primitive chains and $H_{*}(\Omega X)$, Topology 8 (1969), 31-38. MR 38 \#6581.

48. S. Mac Lane, Homology, Academic Press, New York; Springer-Verlag, Berlin, 1963. MR 28 \# 122 .

49. W. Magnus, Beziehungen zwischen Gruppen und Idealen in einem speziellen Ring, Math. Ann. 111 (1935), 259-280.

50. W. S. Massey, Higher order linking numbers, Conf. on Algebraic Topology (Univ. of Illinois at Chicago Circle, 1968), Univ. of Illinois at Chicago Circle, Chicago, 1969, pp. 174-205. MR 40 \#8039.

51. J. P. May, Matric Massey products, J. Algebra 12 (1969), 533-568. MR 39 \#289.

52. J. W. Milnor, Isotopy of links, Algebraic Geometry and Topology, Princeton Univ. Press, Princeton, N. J., 1957, pp. 280-306. MR 19, 1070.

53. N. J., 1963. MR 29 \#634.

54. J. W. Milnor and J. C. Moore, On the structure of Hopf algebras, Ann. of Math. (2) 81 (1965), 211-264. MR 30 \#4259.

55. A. L. Onišcik, On cohomologies of spaces of paths, Mat. Sb. 44 (86) (1958), 3-52; English transl., Amer. Math. Soc. Transl.(2) 25 (1963), 283-334. MR 20 \#6101.

56. A. N. Paršin, A generalization of Jacobian variety, Izv. Akad. Nauk SSSR Ser Mat. 30 (1966), 175-182; English transl., Amer. Math. Soc. Transl. (2) 84 (1969), 187-196. MR 33 \# 4956.

57. R. Ree, Lie elements and an algebra associated with shuffles, Ann. of Math. (2) 68 (1958), 210-220. MR 20 \#6447.

58. ___ Generalized Lie elements, Canad. J. Math. 12 (1960), 493-502. MR 22 \# 5698.

59. J.-P. Serre, Homologie singulière des espaces fibrés. Applications, Ann. of Math. (2) 54 (1951), 425-505. MR 13, 574.

60. L. Smith, Homological algebra and the Eilenberg-Moore spectral sequence, Trans. Amer. Math. Soc. 129 (1967), 58-93. MR 35 \#7337.

61. L_Lectures on the Eilenberg-Moore spectral sequence, Lecture Notes in Math., vol. 134, Springer-Verlag, Berlin and New York, 1970. MR 43 \# 1191.

62. J. W. Smith, The de Rham theorem for general spaces, Tôhoku Math. J. (2) 18 (1966), 115-137. MR 34 \#2027.

63. J. Stallings, Homology and central series of groups, J. Algebra 2 (1965), 170-181. MR 31 \#232.

64. U. Stammbach, Homology in group theory, Springer-Verlag, Berlin, 1973.

65. J. D. Stasheff, H-spaces and classifying spaces-Foundations and recent developments, Algebraic Topology (Proc. Sympos. Pure Math., vol. 22), Amer. Math. Soc., Providence, R. I., 1971, pp. 247-272. MR 47 \#9612.

66. D. Sullivan, Differential forms and the topology of manifolds, Manifolds-Tokyo 1973 (Proc. 
Internat. Conf. on Manifolds, Tokyo, 1973), Math. Soc. Japan, Univ. of Tokyo Press, Tokyo, 1975, pp. 37-49. MR 50 \# 11236.

67. M. E. Sweedler, Hopf algebras, Benjamin, New York, 1969. MR 40 \# 5705.

68. A. Weil, Sur les théorèmes de de Rham, Comment. Math. Helv. 26 (1952), 119-145. MR 14, 307.

69. , Introduction à l'étude des variétés kählériennes (Publ. Inst. Math. Univ. Nancago, VI), Actualités Sci. Ind. No. 1267, Hermann, Paris, 1958. MR 22 \#1921.

70. J. H. C. Whitehead, An expression of Hopf's invariant as an integral, Proc. Nat. Acad. Sci. U.S.A. 33 (1947), 117-123. MR 8, 525.

71. H. Whitney, Geometric integration theory, Princeton Univ. Press, Princeton, N. J., 1957. MR $19,309$.

72. W. T. Wu, Theory of $I^{*}$-functors in algebraic topology, Sci. Sinica 18 (1975), 464-482.

73. W. G. Dwyer, Exotic convergence of the Eilenberg-Moore spectral sequence, Illinois J. Math. 19 (1975), 607-617.

74. V. K. A. M. Gugenheim, On the multiplicative structure of the de Rham theory, J. Differential Geometry 11 (1976), 309-314.

75. __ On Chen's iterated integrals (to appear).

Department of Mathematics, University of Illinois, Urbana, Illinois 61801 